\title{
NUMERICAL UPPER AND LOWER BOUNDS TO THE ULTIMATE \\ LOAD BEARING CAPACITY OF THREE-DIMENSIONAL REINFORCED CONCRETE STRUCTURES
}

\author{
H. Vincent ${ }^{1,2}$, M. Arquier ${ }^{1}$, J. Bleyer ${ }^{2}$, P. de Buhan ${ }^{2}$ \\ ${ }^{1}$ Strains, Paris, France \\ ${ }^{2}$ Laboratoire Navier, UMR 8205, Ecole des Ponts ParisTech, IFSTTAR, CNRS, UPE, \\ Champs-sur-Marne, France \\ Corresponding author: Jérémy Bleyer : jeremy.bleyer@enpc.fr
}

\begin{abstract}
This contribution is addressing the ultimate limit state design of massive three-dimensional reinforced concrete structures based on the finite element implementation of both the upper and lower bound methods of yield design. The strength properties of plain concrete are modelled by means of a tension cut-off Mohr Coulomb condition, while the contribution of the reinforcing bars is taken into account by means of an extended homogenization method. Following a previous article [9], more specifically dedicated to the finite element implementation of the lower bound static approach, the present paper is focused on the upper bound kinematic approach. Similarly to what has been previously done for the lower bound static approach, the reinforced structure is discretized into ten-nodded tetrahedral finite elements, with a quadratic variation of the velocity fields inside each element and velocity jumps across the triangular facets separating any two adjacent elements. This discretization of the velocity fields used in the kinematic approach leads to the formulation of a convex minimization problem which can be solved by resorting to Semi-Definite Programming (SDP) optimization techniques. The whole computational procedure is applied to some illustrative examples, where the implementation of both the static and kinematic methods produces a relatively accurate bracketing of the exact failure load for this kind of structures.
\end{abstract}

Keywords: reinforced concrete structures, yield design, tension cut-off Mohr-Coulomb strength condition, homogenization, upper bound kinematic approach, finite element, semi-definite programming. 


\section{INTRODUCTION}

Assessing the ultimate load bearing capacity of constructions involving massive three-dimensional reinforced concrete components requires a specific analysis, such as the widely acknowledged "strut-and-tie" model (see among many other references [1], [2], [3] or [4]) which can be related to the lower bound static approach of yield design. With a special attention to assessing the ultimate shear capacity of reinforced concrete deep beams, both the lower and upper bound methods of yield design have been implemented in the context of a finite element formulation with the help of linear programming optimization techniques [5]. In the latter study, reinforced concrete was described according to a "mixed modelling" approach, in which plain concrete was modelled as a two-dimensional continuous medium subject to plane stress conditions, while the reinforcement bars were treated as one dimensional flexible beams embedded in the concrete material.

The generalization to the more realistic situation of linear reinforcing inclusions embedded in three-dimensional concrete volumes is posing a somewhat serious difficulty as regards the possibility of treating such a situation by means of a 1D-3D mixed modelling approach. Some attempts to circumvent this problem have already been proposed either in the context of the finite element formulation [6] or making use of an implicit homogenization method [7] or multiphase model [8]. The problem has been quite recently addressed in a previous contribution [9] by resorting to an extended homogenization technique according to which each reinforcing inclusion with its surrounding concrete material is replaced by a homogenized reinforced concrete material. Following this procedure, the lower bound static approach can then be implemented numerically by means of a finite element discretization of the structure leading to a convex optimization problem to be solved by means of a Semi-Definite Programming (SDP) technique [10].

The present contribution concerns the application of the same theoretical model and associated computational procedure, to the kinematic approach of yield design, with the objective to derive rigorous upper bound estimates for the ultimate load bearing capacity of massive three dimensional reinforced concrete structures. The paper is organized as follows.

- The plain concrete strength properties being characterized by means of a tension cut-off Mohr-Coulomb condition, the implementation of the kinematic approach requires the preliminary calculation of its support functions relating to a three dimensional strain rate, as well as to a velocity jump across possible discontinuity surfaces. The expressions of these support functions are also established in the reinforced concrete homogenized zones around the rebars.

- A finite element formulation of the upper bound kinematic approach of yield design is set up based on a discretization of the structure into ten-nodded tetrahedral elements with a quadratic variation of the velocities inside each element and velocity jumps across two adjacent elements. 
- Under such conditions, it is shown that the implementation of the upper bound kinematic approach with such discretized velocity fields amounts to solving a convex minimization problem which can be treated by means of Semi-Definite Programming (SDP) techniques [10], in the same way as for the lower bound static approach to the same kind of problem [9].

The whole computational procedure is first illustrated and validated on the simple example of a uniformly loaded deep beam, where the calculated upper bound estimates are favourably compared to the previously derived lower bound estimates [9]. It is then applied to evaluating the ultimate bearing capacity of a reinforced concrete bridge pier cap subjected to concentrated vertical loads, on the one hand, a circular reinforced concrete foundation slab on the other hand, where in the latter case a semi-analytical design method has already been proposed [11].

\section{THE ADOPTED STRENGTH CRITERIA AND THEIR SUPPORT FUNCTIONS}

Following the approach adopted in [9], a reinforced concrete structure such as the block shown in the left-hand side of figure 1, containing one single inclusion placed along the axis of unit vector $\underline{e}_{1}$, is modelled as a block of same dimensions where the inclusion with the surrounding concrete is replaced by a homogenized reinforced concrete cylindrical zone, as shown in the right hand size of the same figure. The adopted strength conditions in the plain concrete region and the homogenized reinforced concrete zones are the following.

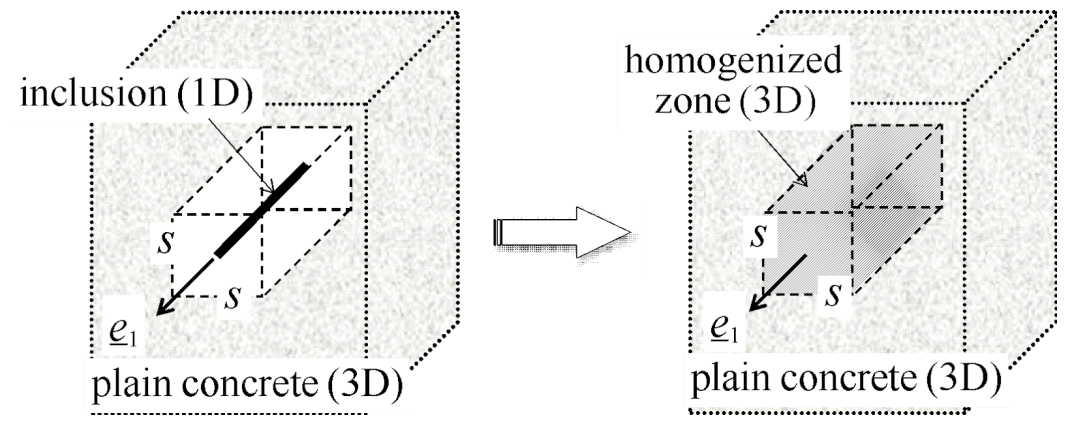

Figure 1. Construction of a homogenized reinforced zone around an individual inclusion of a reinforced concrete block

\subsection{Strength properties of plain concrete: a tension cut-off Mohr Coulomb condition}

The unreinforced or plain concrete is modelled as a 3D homogeneous continuous medium, the strength properties of which are described by means of a tension cut-off Mohr-Coulomb yield condition which may be formulated as:

$$
F^{c}(\underline{\underline{\sigma}})=\sup \left\{K_{p} \sigma_{M}-\sigma_{m}-f_{c} ; \sigma_{M}-f_{t}\right\} \leq 0
$$


where $\sigma_{M}$ and $\sigma_{m}$ represent the major and minor principal stresses, respectively (tensile stresses are counted positive), $f_{t}$ and $f_{c}$ denote the uniaxial tensile and compressive resistances and $K_{p}=(1+\sin \varphi) /(1-\sin \varphi)$ where $\phi$ is the internal friction angle which, in the absence of any complementary information, is usually taken equal to $37^{\circ}\left(K_{p} \approx 4\right)$ [12].

As a preliminary to the implementation of the kinematic approach of yield design, it is necessary to calculate the support functions of this criterion.

$\checkmark$ For a (virtual) strain rate $\underline{d}$, the expression of the support function writes [13]:

$$
\begin{gathered}
\pi^{c}(\underline{\underline{d}})=\sup \left\{\underline{\underline{\sigma}}: \underline{\underline{d}} ; F^{c}(\underline{\underline{\sigma}}) \leq 0\right\} \\
=\left\{\begin{array}{l}
\frac{f_{c}}{2}\left(\sum_{k=1}^{3}\left|d_{k}\right|-\operatorname{tr} \underline{\underline{d}}\right)+\frac{f_{t}}{1-\sin \varphi}\left(\operatorname{tr} \underline{\underline{d}}-\sum_{k=1}^{3}\left|d_{k}\right| \sin \varphi\right) \text { if } \operatorname{tr} \underline{\underline{d}} \geq \sum_{k=1}^{3}\left|d_{k}\right| \sin \varphi \\
+\infty \text { otherwise }
\end{array}\right.
\end{gathered}
$$

where $d_{k}, k=1,2,3$ are the principal strain rate components.

$\boldsymbol{\mathcal { L }}$ Likewise, for a (virtual) velocity jump $\underline{\boldsymbol{V}}$ across any discontinuity surface $\boldsymbol{\Sigma}$ of unit normal $\underline{n}$, the corresponding support function is:

$$
\begin{gathered}
\pi^{c}(\underline{n} ; \underline{V})=\sup \left\{(\underline{\underline{\sigma}} \cdot \underline{n}) \cdot \underline{V} ; F^{c}(\underline{\underline{\sigma}}) \leq 0\right\} \\
=\left\{\begin{array}{l}
\frac{f_{c}}{2}(|\underline{V}|-\underline{V} \cdot \underline{n})+\frac{f_{t}}{1-\sin \varphi}(\underline{\underline{V}} \cdot \underline{n}-|\underline{V}| \sin \varphi) \text { if } \underline{V} \cdot \underline{n} \geq|\underline{V}| \sin \varphi \\
+\infty \text { otherwise }
\end{array}\right.
\end{gathered}
$$

Since:

$$
\pi^{c}(\underline{n} ; \underline{V})=\sup \left\{\underline{T} \cdot \underline{V} ; g^{c}(\underline{T}) \leq 0 \Leftrightarrow \underline{T}=\underline{\underline{\sigma}} \cdot \underline{n} \text { with } F^{c}(\underline{\underline{\sigma}}) \leq 0\right\}
$$

the expression (3) of the latter support function may also be recovered from using the intrinsic curve which represents the strength condition (1) in the Mohr-plane, as shown in figure 2. 


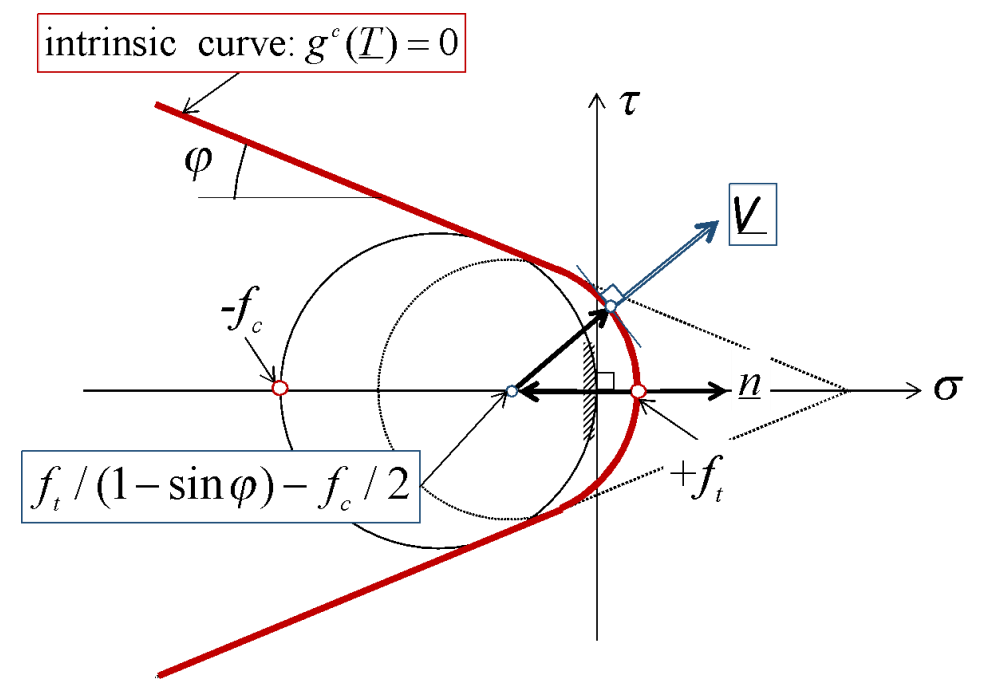

Figure 2. Intrinsic curve of the plain concrete strength condition in the Mohr-plane and calculation of the support function relative to a velocity discontinuity

\subsection{Reinforced concrete: a homogenized strength condition}

The (anisotropic) strength criterion in the homogenized reinforced zone defined around each reinforcing bar is the following (see [9] and [14] for more details):

$$
F^{r c}(\underline{\underline{\sigma}}) \leq 0 \Leftrightarrow\left\{\begin{array}{l}
\underline{\underline{\sigma}}=\underline{\underline{\sigma}}^{c}+\sigma^{r} \underline{e}_{1} \otimes \underline{e}_{1} \\
\text { with } \quad F^{c}\left(\underline{\underline{\sigma}}^{c}\right) \leq 0 \text { and }-k \sigma_{0} \leq \sigma^{r} \leq \sigma_{0}
\end{array}\right.
$$

where $0 \leq k \leq 1$ is a non-dimensional factor accounting for a reduction of the bar compressive strength due to potential buckling, $\underline{e}_{1}$ is the unit vector parallel to the reinforcing bar, and $\sigma_{0}$ is defined as the tensile resistance of these bars per unit transverse area:

$$
\sigma_{0}=n_{0} / s^{2}
$$

where $n_{0}$ denotes the tensile resistance of each individual reinforcing bar, while $s$ is the side of the homogenized reinforced zone (Figure 1), so that :

$$
\sigma_{0}=A^{s} f_{y}^{s} / s^{2}=\eta f_{y}^{s}
$$

In the above equation, $f_{y}^{s}$ denotes the uniaxial strength of the bar constituent material (steel for example) and $A^{s}$ the bar cross-sectional area, so that $\eta$ represents the reinforcement volume fraction in the homogenized zone. 
It is to be recalled that the validity of the above macroscopic strength criterion (5) is subject to the verification of two important conditions (see [14] for more details):

- The reinforcing bars are perfectly bonded to the surrounding concrete material, which means for instance that no slippage occurs at the bar/concrete interface.

- The reinforcement volume fraction remains sufficiently small $(\eta<<1)$, whereas the resistance of the reinforcing material is much higher than that of the concrete material and notably its tensile strength $\left(f_{y}^{s}>>f_{c}\right)$.

The support functions of the macroscopic strength condition (5) may be easily calculated as follows:

$$
\begin{gathered}
\pi^{r c}(\underline{\underline{d}})=\sup \left\{\underline{\underline{\sigma}}: \underline{\underline{d}} ; F^{r c}(\underline{\underline{\sigma}}) \leq 0\right\} \\
=\sup \left\{\underline{\underline{\sigma}}^{c}: \underline{\underline{d}} ; F^{c}\left(\underline{\underline{\sigma}}^{c}\right) \leq 0\right\}+\sup \left\{\left(\sigma^{r} \underline{\underline{e}}_{1} \otimes \underline{\underline{e}}_{1}\right): \underline{\underline{d}} ;-k \sigma_{0} \leq \sigma^{r} \leq+\sigma_{0}\right\} \\
=\pi^{c}(\underline{\underline{d}})+\sigma_{0} \sup \left\{-k d_{11}, d_{11}\right\}
\end{gathered}
$$

and

$$
\begin{gathered}
\pi^{r c}(\underline{n} ; \underline{V})=\sup \left\{(\underline{\underline{\sigma}} \cdot \underline{n}) \cdot \underline{V} ; F^{r c}(\underline{\underline{\sigma}}) \leq 0\right\} \\
=\sup \left\{\left(\underline{\underline{\sigma}}^{c} \cdot \underline{n}\right) \cdot \underline{V} ; F^{c}\left(\underline{\underline{\sigma}}^{c}\right) \leq 0\right\}+\sup \left\{\sigma^{r}\left(\underline{e}_{1} \cdot \underline{n}\right) \underline{\underline{e}}_{1} \cdot \underline{V} ;-k \sigma_{0} \leq \sigma^{r} \leq+\sigma_{0}\right\} \\
=\pi^{c}(\underline{n} ; \underline{\underline{V}})+\sigma_{0} \sup \left\{-k n_{1} V_{1}, n_{1} V_{1}\right\}
\end{gathered}
$$

\subsection{Implementing the upper bound kinematic approach}

This approach is, as usual, derived from the dualization of the static approach through the application of the virtual work principle [13]. Assuming for instance, without any loss of generality, that the reinforced concrete structure is subject to one single loading parameter $Q$, and given any kinematically admissible (K.A.) virtual velocity field $\underline{U}$, the virtual work of external forces may be put in the following form:

$$
P_{e}(\underline{U})=Q q(\underline{U})
$$

while the maximum resisting work developed in the same velocity field is computed by using the previously calculated support functions:

$$
P_{m r}(\underline{U})=\int_{\Omega^{c}} \pi^{c}(\underline{\underline{d}}) \mathrm{d} \Omega^{c}+\int_{\Omega^{r c}} \pi^{r c}(\underline{\underline{d}}) \mathrm{d} \Omega^{r c}+\int_{\Sigma^{c}} \pi^{c}(\underline{n} ; \underline{\underline{V}}) \mathrm{d} \Sigma^{c}+\int_{\Sigma^{r c}} \pi^{r c}(\underline{n} ; \underline{V}) \mathrm{d} \Sigma^{r c}
$$

with $\Omega^{c}$ (respectively $\Sigma^{c}$ ) denoting the part of the structure occupied by the plain concrete material (respectively the velocity jump surfaces in $\Omega^{c}$ ) and $\Omega^{r c}$ (respectively $\Sigma^{r c}$ ) the set of 
all the homogenized reinforced concrete zones (resp. the velocity jump surfaces in $\Omega^{r c}$ ). Making use of (8) and (9), the expression of the maximum resisting work may rewritten as:

$$
\begin{aligned}
P_{m r}(\underline{U}) & =\int_{\Omega} \pi^{c}(\underline{\underline{d}}) \mathrm{d} \Omega+\int_{\Sigma} \pi^{c}(\underline{n} ; \underline{V}) \mathrm{d} \Sigma \\
& +\int_{\Omega^{r c}} \sigma_{0} \sup \left\{-k d_{11}, d_{11}\right\} \mathrm{d} \Omega^{r c}+\int_{\Sigma^{r c}} \sigma_{0} \sup \left\{-k n_{1} V_{1}, n V_{1}\right\} \mathrm{d} \Sigma^{r c}
\end{aligned}
$$

where $\Omega=\Omega^{c} \cup \Omega^{r c}$.

Under these conditions, the upper bound kinematic theorem of yield design states that the ultimate load bearing capacity of the structure, denoted by $Q^{+}$, satisfies the following inequality, valid for any kinematically admissible velocity field:

$$
\forall \underline{U} \text { K.A. , } Q^{*} q(\underline{U}) \leq P_{r m}(\underline{U})
$$

thus leading to the following upper bound value for $Q^{+}$:

$$
\forall \underline{U} \text { K.A. } ; q(\underline{U})>0, Q^{+} \leq Q^{\mathrm{ub}}=P_{r m}(\underline{U}) / q(\underline{U})
$$

As it is clearly apparent from (14), obtaining the best upper bound estimate to $Q^{+}$amounts to solving a minimization problem over the set of K.A. velocity fields, which in the present case will be achieved through a finite element discretization of the structure in much the same way as for the lower bound static approach [9].

\section{NUMERICAL IMPLEMENTATION OF THE UPPER BOUND KINEMATIC APPROACH [14]}

Similarly to the lower bound static approach which leads to a maximization problem and can be treated through SDP techniques (see [9]), the upper bound kinematic approach (14) is associated with a minimization problem which can also be solved by means of Semidefinite Programming, as briefly explained in the sequel.

\subsection{Formulation of the support functions as SDP minimization problems}

We start from the Mohr-Coulomb strength criterion (i.e. without the tension cut-off condition):

$$
F^{c}(\underline{\underline{\sigma}})=F^{M C}(\underline{\underline{\sigma}})=K_{p} \sigma_{M}-\sigma_{m}-f_{c} \leq 0
$$


which may be rewritten in terms on linear matrix inequalities as (see Eq. (18) of [9]):

$$
\underline{\underline{\sigma}}-K_{p}^{-1} t_{m} \underline{\underline{1}} \leq K_{p}^{-1} f_{c \underline{\underline{1}}} \text { and } t_{m \underline{\underline{1}}}-\underline{\underline{\sigma}} \leq 01
$$

where $t_{m}$ is an auxiliary variable. It follows that the corresponding support function may be calculated as follows:

$$
\begin{gathered}
\pi^{M C}(\underline{\underline{d}})=\sup \left\{\underline{\underline{\sigma}}: \underline{\underline{d}} ; F^{M C}(\underline{\underline{\sigma}}) \leq 0\right\} \\
=\sup _{\left(\underline{\underline{\sigma}}, t_{m}\right)}\left\{\underline{\underline{\sigma}}: \underline{\underline{d}} ; \underline{\underline{\sigma}}-K_{p}^{-1} t_{m} \underline{1} \leq K_{p}^{-1} f_{c} \underline{\underline{1}} \text { and }-\underline{\underline{\sigma}}+t_{m} \underline{\underline{1}} \leq 0\right\}
\end{gathered}
$$

Let us introduce the Lagrangian of this maximization problem defined as:

$$
\begin{aligned}
L\left(\underline{\underline{\sigma}}, t_{m}, \underline{\underline{e}}_{1}, \underline{\underline{e}}_{2}\right) & =\underline{\underline{\sigma}}: \underline{\underline{d}}+\left(-\underline{\underline{\sigma}}+K_{p}^{-1} t_{m} \underline{\underline{1}}+K_{p}^{-1} f_{c} \underline{1}\right): \underline{\underline{e}}_{1}+\left(\underline{\underline{\sigma}}-t_{m}\right): \underline{\underline{e}}_{2} \\
& =\underline{\underline{\sigma}}:\left(\underline{\underline{d}}-\underline{\underline{e}}_{1}+\underline{\underline{e}}_{2}\right)+t_{m}\left(K_{p}^{-1} \operatorname{tr}\left(\underline{\underline{e}}_{1}\right)-\operatorname{tr}\left(\underline{\underline{e}}_{2}\right)\right)+f_{c} \operatorname{tr}\left(\underline{\underline{e}}_{1}\right)
\end{aligned}
$$

with its partial derivatives:

$$
\partial L / \partial \underline{\underline{\sigma}}=\underline{\underline{d}}-\underline{\underline{e}}_{1}+\underline{\underline{e}}_{2}=0 ; \partial L / \partial t_{m}=K_{p}^{-1} \operatorname{tr}\left(\underline{\underline{e}}_{1}\right)-\operatorname{tr}\left(\underline{\underline{e}}_{2}\right)=0
$$

where $\left(\underline{\underline{e}}_{1}, \underline{\underline{e}}_{2}\right)$ are the Lagrange multipliers associated with the two SDP constraints of (17), which must verify $\underline{\underline{e}}_{1}, \underline{\underline{e}}_{2} \succeq 0$.

Finally, the support function can be computed as the result of the following minimization problem involving two linear constraints and two SDP constraints:

$$
\pi^{M C}(\underline{\underline{d}})=\inf _{\left(\underline{\underline{e}}_{1}, \underline{\underline{e}}_{2}\right)}\left\{\begin{array}{c}
\underline{\underline{d}}-\underline{\underline{e}}_{1}+\underline{\underline{e}}_{2}=0 \\
f_{c} \operatorname{tr}\left(\underline{\underline{e}}_{1}\right) ; K_{p}^{-1} \operatorname{tr}\left(\underline{\underline{e}}_{1}\right)-\operatorname{tr}\left(\underline{\underline{e}}_{2}\right)=0 \\
\underline{\underline{e}}_{1} \geq 0, \underline{\underline{e}}_{2} \geq 0
\end{array}\right\}
$$

Following the same kind of approach, analogous SDP formulations are obtained for the support function of the tension cut-off Mohr-Coulomb criterion (1), as well as for that of the macroscopic strength condition of reinforced concrete (5). For instance the support function (2) of the tension cut-off (or truncated) Mohr-Coulomb criterion adopted here for describing the strength properties of plain concrete, can be calculated as (see [10],[15] for more details):

\footnotetext{
${ }^{1}$ Where
} 


$$
\pi^{c}(\underline{\underline{d}})=\pi^{T M C}(\underline{\underline{d}})=\inf _{\left(\underline{\underline{e}}_{1}, \underline{\underline{e}}_{2}\right)}\left\{\begin{array}{cc}
\underline{\underline{d}}-\underline{\underline{e}}_{1}+\underline{\underline{e}}_{2}=0, \operatorname{tr}\left(\underline{\underline{e}}_{2}\right)-s_{1}-s_{2}=0 \\
S_{1} f_{t}+s_{2} f_{c} ; \\
K_{p}^{-1} s_{2}-\operatorname{tr}\left(\underline{\underline{e}}_{2}\right)=0 \\
\underline{\underline{e}}_{1}, \underline{\underline{e}}_{2} \geq 0, s_{1}, s_{2} \geq 0
\end{array}\right\}
$$

where $s_{1}$ and $s_{2}$ are two additional Lagrange multipliers associated with the tension cut-off condition.

\section{2. $3 D$ finite element discretization}

In a similar way as it has been done for the lower bound static approach in [9], the 3D geometrical domain $\Omega$ occupied by the reinforced concrete structure is discretized into $N_{e}$ ten-node tetrahedral finite elements $\Omega_{e}$, as shown in Figure 3. The virtual velocity fields used in the kinematic approach are quadratic functions of the position $\underline{x}$ inside each element, so that they can be expressed as:

$$
\forall \underline{x} \in \Omega_{e}, \underline{U}(\underline{x})=\sum_{k=1}^{10} N^{k}(\underline{x}) \underline{U}^{k}
$$

where $\underline{U}(\underline{x})$ is the (virtual) velocity vector at point $\underline{x}, \underline{U}^{k}$ is the velocity at node number $k$ of the element and $N^{k}(\underline{x}), k=1, \ldots, 10$ are the quadratic interpolation functions. It should be noted that the considered velocity fields may be discontinuous across the triangular facets separating two adjacent elements.

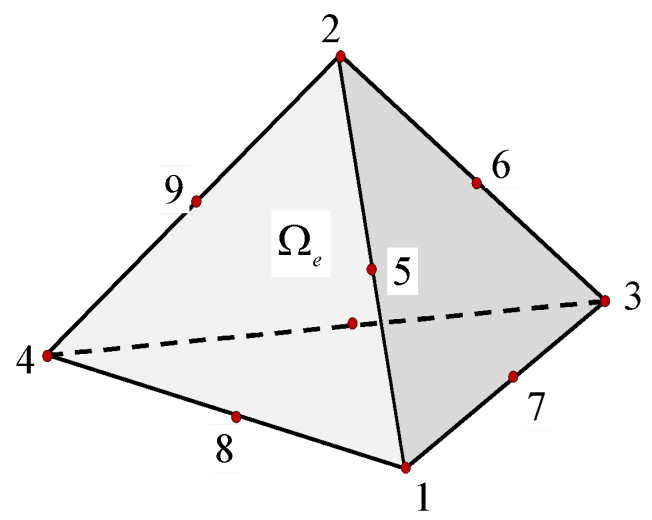

Figure 3. Ten-node tetrahedral finite element used in the discretization of the reinforced concrete structure 
The maximum resisting work developed in such a piecewise quadratic velocity field is evaluated from integrating the support functions over each element, then by summation over all such elements. Referring for instance to the simplest configuration of a structure made of plain concrete ( $\Omega=\Omega^{c}$ ) and to a continuous velocity field discretized according to $(21)$, the total maximum resisting work is approximated as follows:

$$
P_{m r}(\underline{U})=\int_{\Omega=\Omega^{c}} \pi^{c}(\underline{\underline{d}}) \mathrm{d} \Omega=\sum_{e=1}^{N_{e}} \int_{\Omega_{e}} \pi^{c}(\underline{\underline{d}}) \mathrm{d} \Omega_{e} \leq \sum_{e=1}^{N_{e}} \sum_{i=1}^{4} \omega_{i} J_{e}\left(s_{1}^{i} f_{t}+s_{2}^{i} f_{c}\right)
$$

where $N_{e}$ is the number of finite elements and $J_{e}$ the Jacobian determinant of the transformation between the reference element and the real one. As shown by the above expression, the maximum resisting work relating to each tetrahedral element $\Omega_{e}$ is evaluated by excess through the use of four integration points (with weighting coefficients $\omega_{i}, i=1, \ldots, 4$ ) located at the vertices of the element, thus preserving the upper bound status of the numerical evaluation associated with the right hand member [15]. The generalization to the situation of reinforced concrete zones on the one hand, the presence of velocity discontinuities between adjacent elements on the other hand, is explained in more details in [15].

Finally, according to (14), the best numerical upper bound estimate of $Q^{+}$is obtained as the solution of a Semidefinite Programming optimization problem making use of a dedicated solver [16].

\section{IMPLEMENTATION ON THREE ILLUSTRATIVE TEST EXAMPLES}

\subsection{Reinforced concrete beam under uniform loading}

A first application of the above described upper bound numerical method is now performed on the same example as that previously analysed with the numerical lower bound static approach in [9]. The main geometrical characteristics of the problem are recalled in Figure 4 below. 


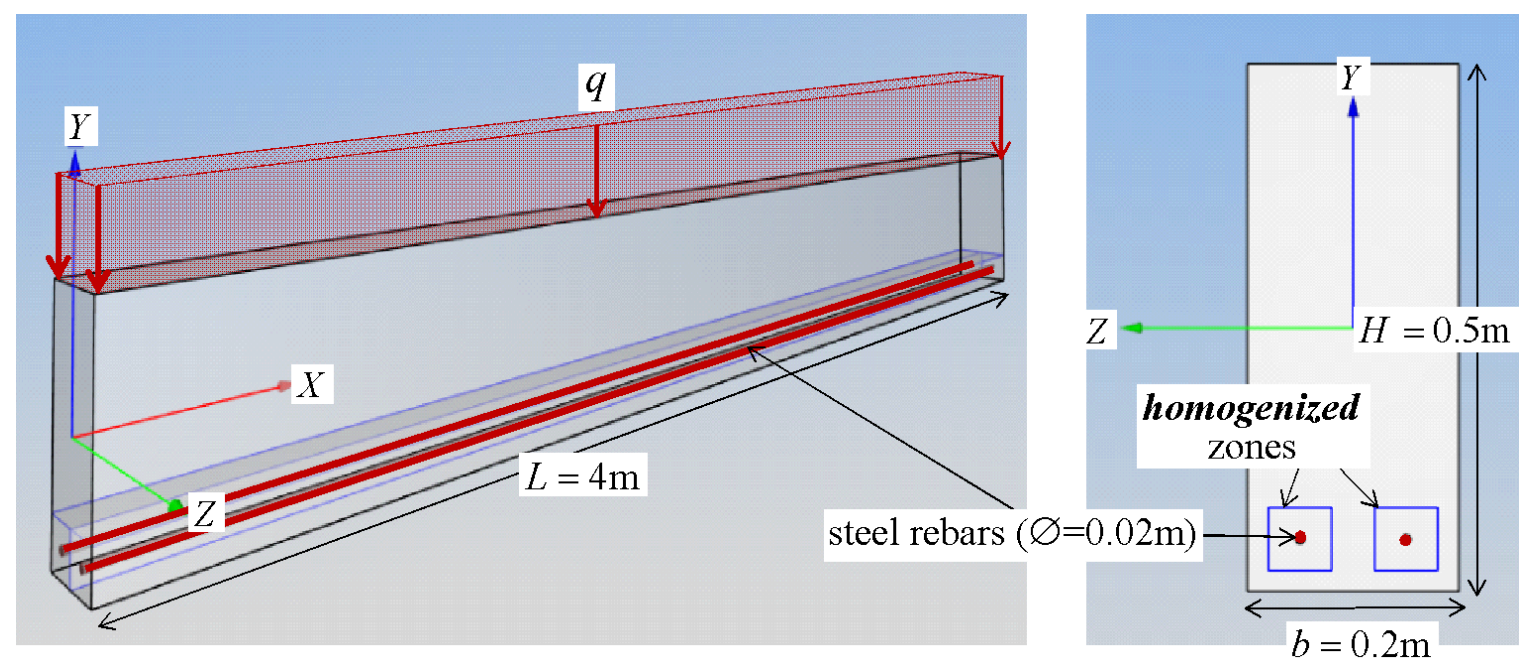

Figure 4. Reinforced concrete beam under uniform loading

The beam is made of a homogeneous concrete material, with the following uniaxial tensile and compressive strength characteristics:

$$
f_{t}=0.5 \mathrm{MPa} \text { and } f_{c}=40 \mathrm{MPa}
$$

and an internal friction angle $\phi$ is taken equal to $37^{\circ}$. It is reinforced by two longitudinal rebars of diameter equal to $2 \mathrm{~cm}$, made of a steel with a uniaxial strength equal to $f_{y}^{s}=400 \mathrm{MPa}$. They are located at the lower part of the beam cross section as shown in Fig. 4.

The beam-like structure is subjected to a uniform surface loading $q$ applied on its top surface. Its left end $(X=0)$ is perfectly clamped (velocity vector equal to zero: $\underline{U}=0$ ), while the boundary conditions at its right end $(X=L)$ are:

$$
T_{x}=\sigma_{x x}=0, U_{y}=U_{z}
$$

It is to be noted that the above $3 \mathrm{D}$ conditions correspond, in the framework of a 1D-beam modelling of the structure, to a simple support free to move horizontally along the $X$-axis and rotate about the $Y$-axis (axial force $N$ and bending moment $M$ being set equal to zero).

All the results obtained from the implementation of both the numerical static and kinematic methods have been gathered in Figure 5 for a beam of length equal to $4 \mathrm{~m}$. The corresponding lower and upper bounds have been calculated for three different sizes of the homogenized zones $(0.06 \mathrm{~m} \times 0.06 \mathrm{~m}, 0.08 \mathrm{~m} \times 0.08 \mathrm{~m}$ and $0.10 \mathrm{~m} \times 0.10 \mathrm{~m})$ and increasing number of finite 
elements along the horizontal axis. The horizontal solid line placed between the calculated upper and lower bounds represents the evaluation of the ultimate load bearing capacity of the structure modelled as a 1D-beam element [9].

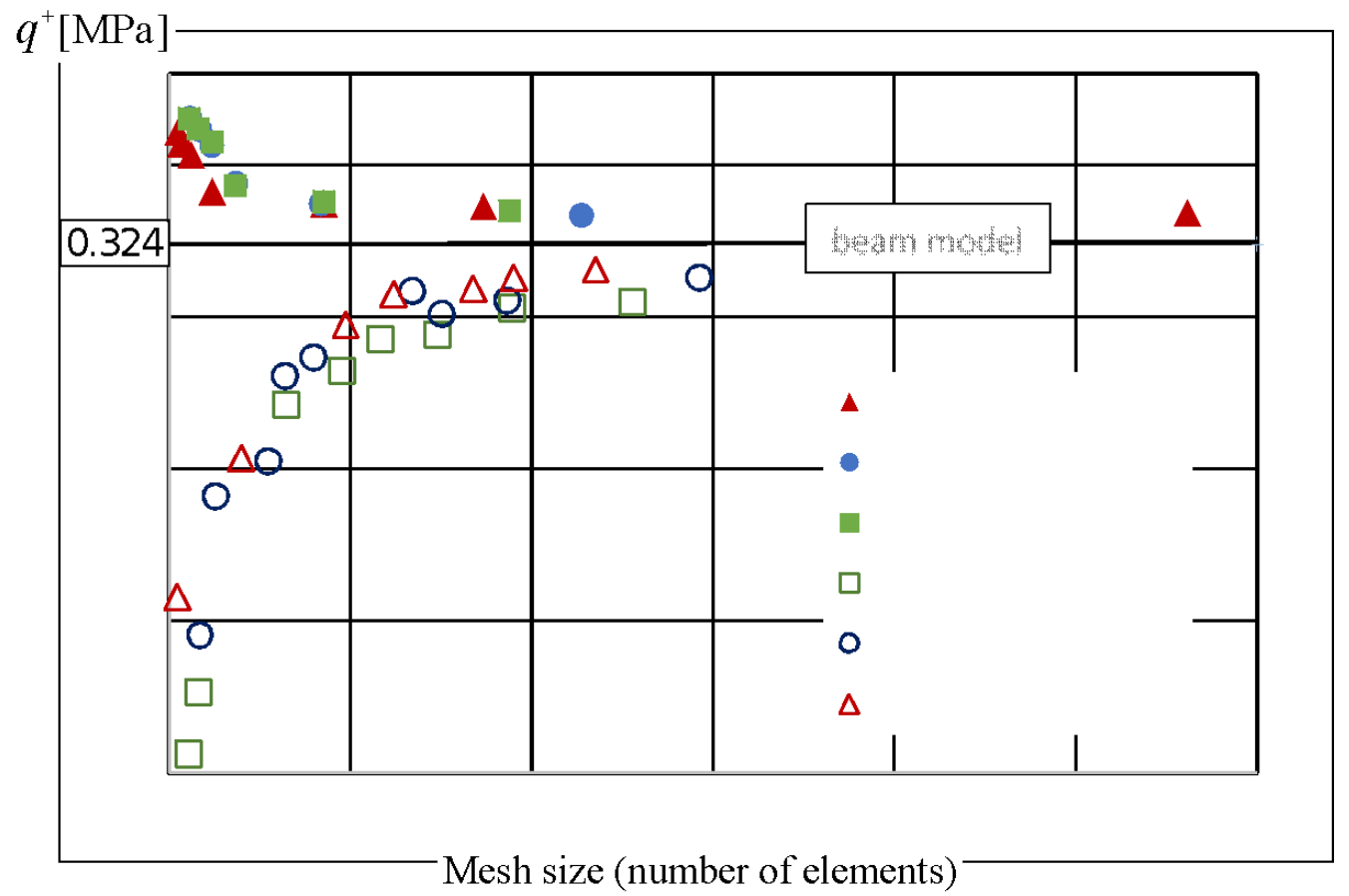

Figure 5. Numerical upper and lower bound estimates as functions of the homogenized zones size and number of finite elements for a $4 \mathrm{~m}$-long reinforced concrete beam.

As can be seen from this figure, the upper (respectively lower) bound estimate is decreasing (resp. increasing) with respect to the size of the finite element mesh, while the size of the homogenized reinforced zones has less and less influence on the bounds as the size of the mesh is increasing. The optimal bounds have been reported in Table I below, which gives evidence of a very good agreement with the result of the 1D-beam analytical model detailed in [9], as well as a fairly accurate bracketing of the exact ultimate load.

\begin{tabular}{|c|c|c|c|}
\hline $\begin{array}{c}\text { Size of homogenized reinforced } \\
\text { zones }\left(\mathrm{m}^{2}\right)\end{array}$ & $0.1 \times 0.1$ & $0.08 \times 0.08$ & $0.06 \times 0.06$ \\
\hline Analytical: $q_{1 D}^{+}(\mathrm{MPa})$ & 0.324 & 0.324 & 0.324 \\
\hline$q_{3 D}^{\mathrm{lb}}(\mathrm{MPa})$ & 0.316 & 0.313 & 0.305 \\
\hline
\end{tabular}




\begin{tabular}{|c|c|c|c|}
\hline$q_{3 D}^{\mathrm{ub}}(\mathrm{MPa})$ & 0.334 & 0.334 & 0.335 \\
\hline$\Delta(\%)$ & \pm 3 & \pm 3 & \pm 4.5 \\
\hline
\end{tabular}

Table I. Optimal lower and upper bounds on the ultimate load bearing capacity of the $4 \mathrm{~m}$-long reinforced concrete beam and comparison with analytical approach.

Figure 6 displays the numerically optimized velocity and stress fields for the $4 \mathrm{~m}$-long longitudinally reinforced concrete beam. The results of the upper bound kinematic approach have been obtained for a mesh containing 17332 elements. Figures 5(a) and (b) represent the optimal velocity field and strain rate distribution, respectively. The corresponding failure mechanism involves two "plastic hinges" where the strain rate distribution is concentrated: the first one at the clamped left end of the beam, the other one around the middle section of the beam.

The optimized stress field resulting from the application of the numerical lower bound static approach [9] with a mesh of 23552 finite elements.is shown in Figure 5(c), where the principal components of stress, along with their orientation, are represented at any point of the mesh. Such an optimized stress field is of obviously reminiscent of classically used strut-and-tie models of reinforced concrete structures, with two inclined lateral compressive "struts" in the concrete connected to a bottom horizontal tensile "tie" in the reinforced concrete region.

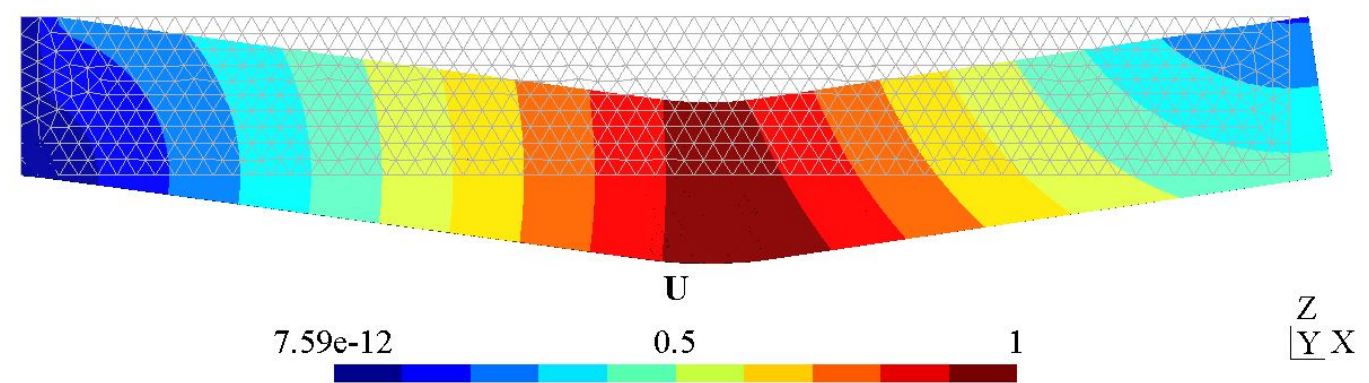

(a) 

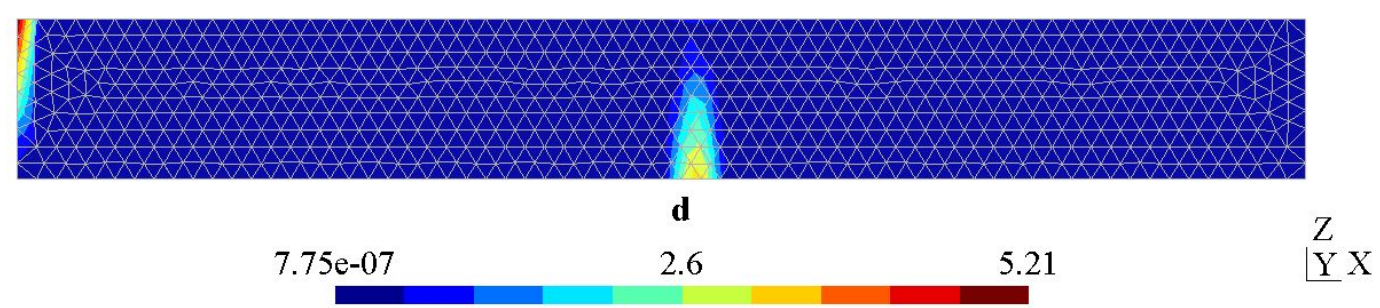

(b)

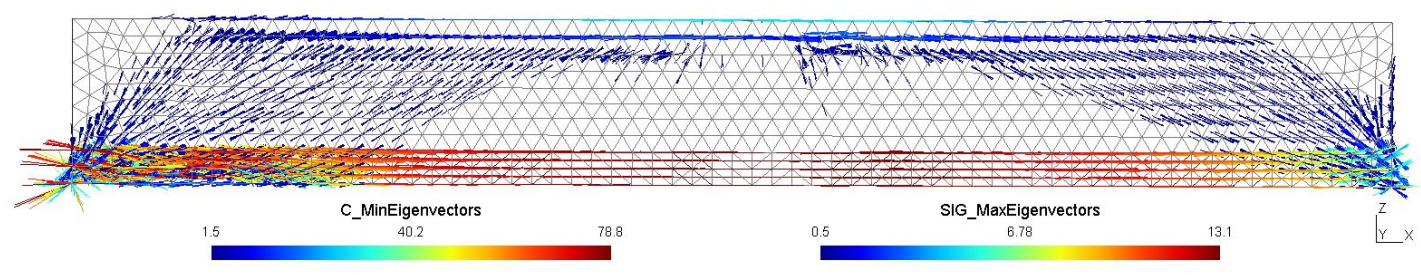

(c)

Figure 6. Optimal velocity field (a), strain rate distribution (b) and stress field (c) for a 4m-long reinforced concrete beam at failure

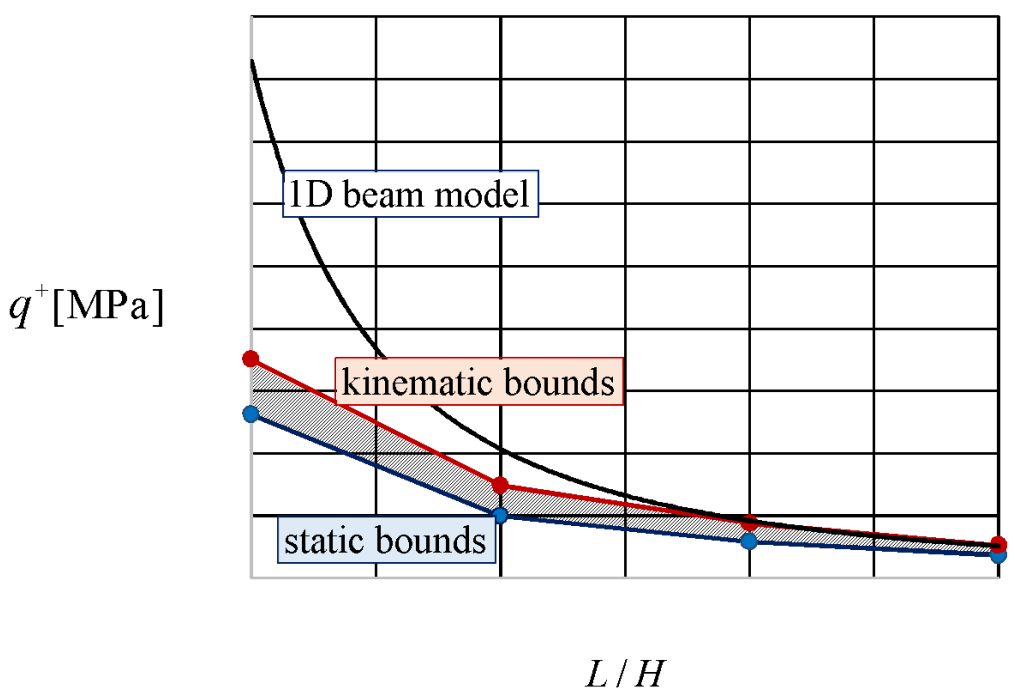

Figure 7. Comparison between the 1D analytical and 3D numerical estimates as a function of the reinforced concrete beam slenderness

The curves drawn in Figure 7 represent the variations of the 3D finite element bounds and the analytical estimate derived from a 1D beam model, equal to [9]:

$$
q_{1 \mathrm{D}}^{+}(\mathrm{MPa})=\frac{5.18}{L^{2}}
$$


(where $L[\mathrm{~m}]$ is the beam length, the depth $H=0.5 \mathrm{~m}$ and thickness $b=0.2 \mathrm{~m}$ being kept constant), as functions of the beam slenderness $L / H$ ranging between 0.5 and 2 , that is for "short" or "deep" beams. It clearly appears from this graph that the $1 \mathrm{D}$ analytical value (18) overestimates the actual value of the ultimate load which is comprised between the $3 \mathrm{D}$ upper and lower bounds. This is obviously due to the fact that the 1D-model, which is based on the notion of interaction diagram, does not take the limited shear strength capacities of the beam into account.

\subsection{Compression of plain concrete block}

In order to further validate the above described numerical procedure, at least for plain concrete structures, the comparison will now be made with the results obtained by [9] on the unconfined compression problem of a homogeneous concrete block, making use of the same numerical methods as that described in the paper. A perspective view of the entire square parallelepipedic block is shown in Figure 8(a). The block is submitted to a vertical compressive force applied onto both its lower and upper horizontal faces by means of rigid plates which are perfectly adherent to the faces.

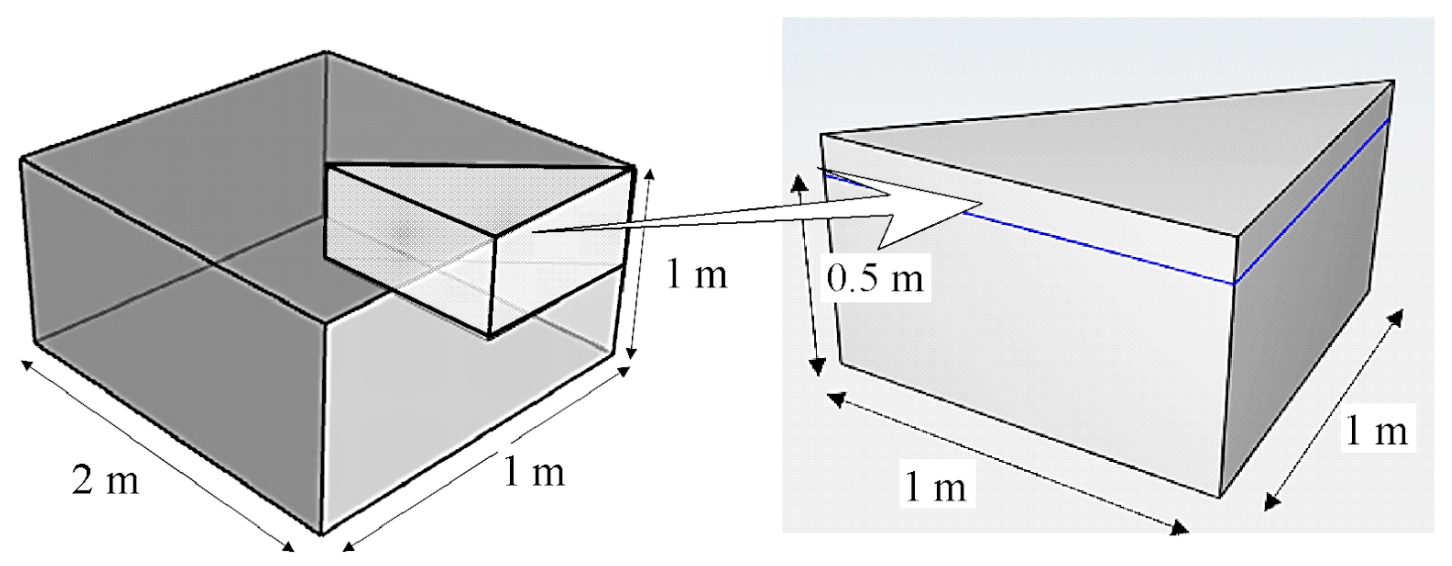

(a)

(b)

Figure 8 . Homogeneous concrete block (a) with its $1 / 16^{\text {th }}$ part (b)

Thanks to the obvious symmetries of the problem with respect to the horizontal and vertical middle planes, the problem can be reduced to that of the $1 / 16^{\text {th }}$ part of the structure shown in Figure $8(\mathrm{~b})$ with the following appropriate conditions:

$\checkmark$ uniform pressure $p$ applied on top of the rigid plate; 
$\checkmark$ stress-free lateral side of the block;

$\checkmark$ the three remaining sides are in smooth contact with the fixed corresponding symmetry planes.

The compressive strength of the block $p^{+}$is evaluated by using both the numerical upper and lower bound methods with a sufficiently refined regular mesh made of tetrahedral elements. The homogenous constituent material obeys a Mohr-Coulomb strength condition with the same characteristics as those adopted in [9]: friction angle equal to $\varphi=30^{\circ}$ and uniaxial compressive strength equal to $f_{c}=40 \mathrm{MPa}$.

It is to be noted that in the case when the pressure is directly applied to the block or when the contact between the rigid plate and the block is smooth, the solution to this problem is immediate. It corresponds to a homogeneous uniaxial compressive stress field as regards the static approach and a homogenous strain rate field for the kinematic approach, leading to the exact limit load value, which nothing but the uniaxial compressive strength of the material: $q^{+}=f_{c}=40 \mathrm{MPa}$.

Figure 9(a) represents the optimal velocity field (failure mechanism) and Figure 9(b) the optimized stress field (principal stresses) relative to the problem of perfectly adherent loading plates, leading to the following optimized lower and upper bounds:

$$
p^{\text {lb }}=95.1 \mathrm{MPa} \leq p^{+} \leq p^{\text {ub }}=110.3 \mathrm{MPa}
$$

or in non-dimensional form:

$$
2.37 \leq p^{+} / f_{c} \leq 2.75
$$




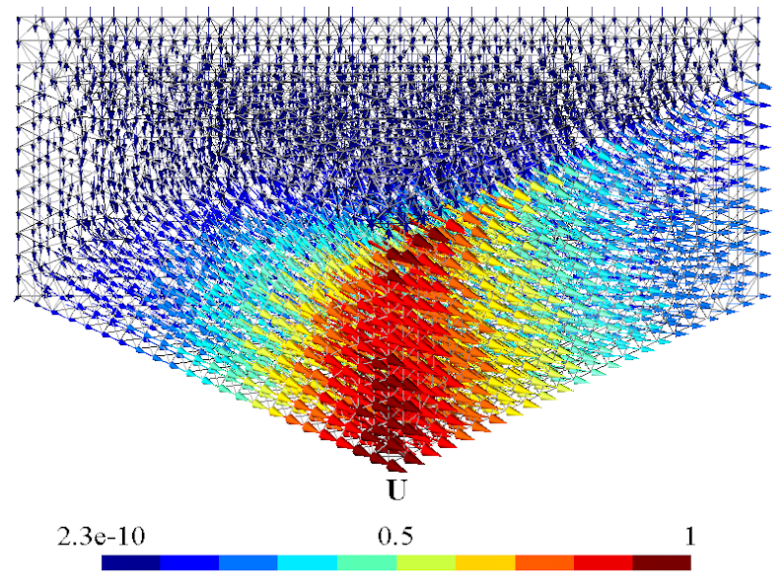

(a)

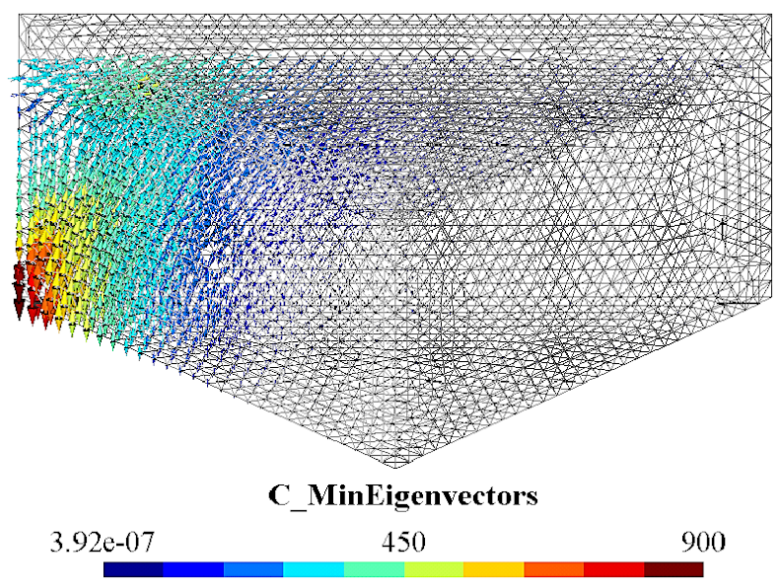

(b)

Figure 9. Optimal failure mechanism (a) and stress field (b)

Our results are now compared with those obtained by Martin and Makrodimopoulos [9] on exactly the same problem, which are expressed as follows:

$$
8.35 \leq p^{+} / C \leq 10.06
$$

where $C$ is the material cohesion which is related to the its uniaxial compressive strength by the classical equation:

$$
f_{c}=\frac{2 C \cos \varphi}{1-\sin \varphi} \cong 3.46 C
$$

so that (27) can be rewritten as:

$$
2.41 \leq p^{+} / f_{c} \leq 2.91
$$

showing from comparing with (26) that our results are very close to those of [9].

Remark. In spite of its apparent simplicity, the problem at stake remains a challenging issue as concerns the efficiency of the finite element formulation of yield design approaches in providing accurate bounds, since in both cases the relative discrepancy between the bounds remains significant: $18.5 \%$ in (29) instead of $14.5 \%$ in (26). Of course, the use of a mesh refinement procedure, would probably help improving the previous bounds.

\subsection{Ultimate bearing capacity of plain concrete bridge pier cap}

A third example of genuinely three-dimensional structure, where the present approach will be compared with otherwise available results, is the case of a bridge pier cap made, once again, of unreinforced plain concrete, as shown in Figure 10. Such a relatively complex 
three-dimensional structure is simply modelled here as a $3 \times 3 \times 1.5 \mathrm{~m}^{3}$ parallelepipedic block. It is vertically loaded by means of four $0.7 \times 0.7 \mathrm{~m}^{2}$ square rigid pads place on its upper surface, while rigidly connected on its base to a rectangular $1.5 \times 0.7 \mathrm{~m}^{2}$ bridge pier. The constituent material is a plain concrete, obeying a tension cut-off Mohr-Coulomb (TMC) strength condition with $f_{c}=40 \mathrm{MPa}$ and $f_{t}=0.5 \mathrm{MPa}$. Once again, due to the symmetries of the problem with respect to the vertical middle-planes, the calculations are performed on a quarter part of the structure.
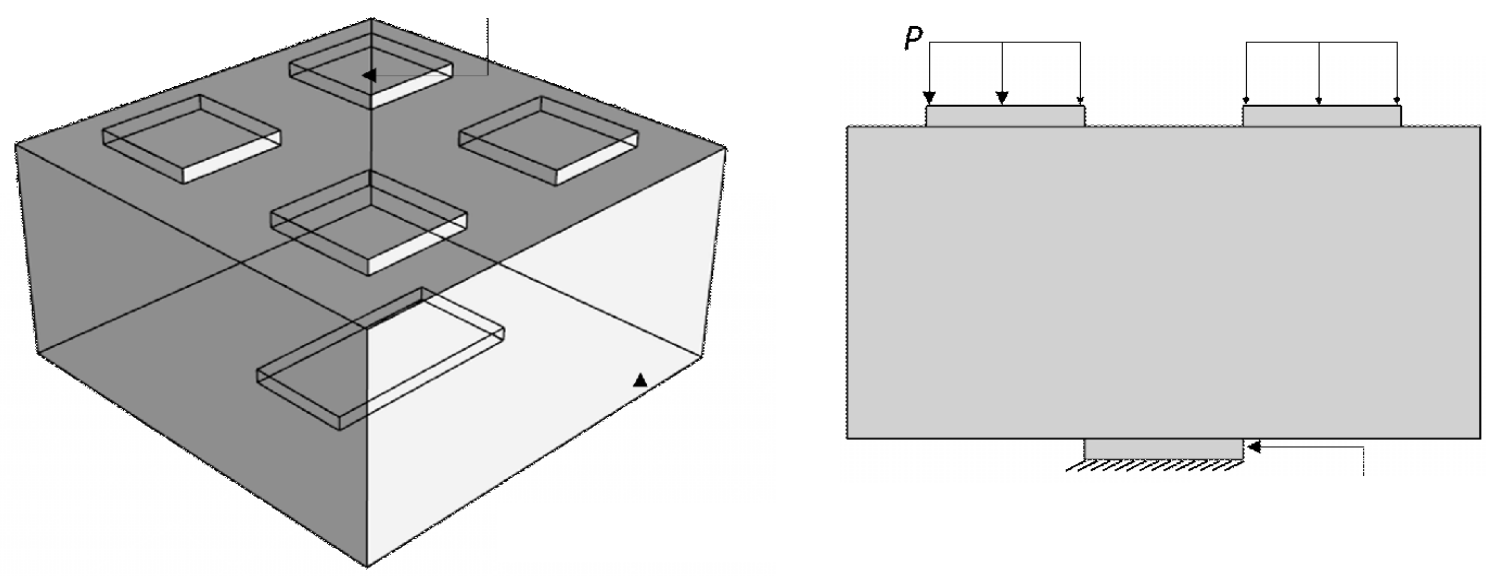

Figure 10. Perspective and side views of a vertically loaded unreinforced bridge pier cap

\subsubsection{Results and comparison with the OptumG3 finite element code}

Table II below gives the optimized numerical bounds on the ultimate load $P^{+}$calculated with our specific computer code and from using the available finite element code OptumG3 [17] quite recently developed for dealing with three-dimensional limit analysis/yield design problems.

\begin{tabular}{|c|c|c|c|c|}
\cline { 2 - 5 } \multicolumn{1}{c|}{} & $P^{\mathrm{lb}}(\mathrm{MPa})$ & $P^{\mathrm{ub}}(\mathrm{MPa})$ & $\left(P^{\mathrm{lb}}+P^{\mathrm{ub}}\right) / 2(\mathrm{MPa})$ & $\Delta(\%)$ \\
\hline OptumG3 & 3.85 & 4.38 & 4.11 & \pm 6.4 \\
\hline
\end{tabular}




\begin{tabular}{|l|l|l|l|l|}
\hline Present approach & 3.89 & 4.46 & 4.17 & \pm 6.8 \\
\hline
\end{tabular}

Table II. Upper and lower bounds calculated by the OptumG3 software and the present approach

Taking in each case the average value of the upper and lower bounds, the ultimate load is equal to $4.17 \mathrm{MPa} \pm 6.8 \%$ according to the present approach and to $4.11 \mathrm{MPa} \pm 6.4 \%$ when using the OptumG3 code. As a consequence, a quite good agreement is observed between the two calculated estimates, since the relative difference is less than $1.5 \%$. Adopting the same mesh refinement procedure as that used in Optum G3, the result of the present approach can be further improved, leading to the new estimated value of $4.15 \mathrm{MPa} \pm 3.5 \%$. Figure 11(a) allows to visualize the corresponding optimal failure mechanism of the structure and Figure 11(b) represents the optimal stress field clearly showing four compressive struts converging from the loading pads to the supporting pier.

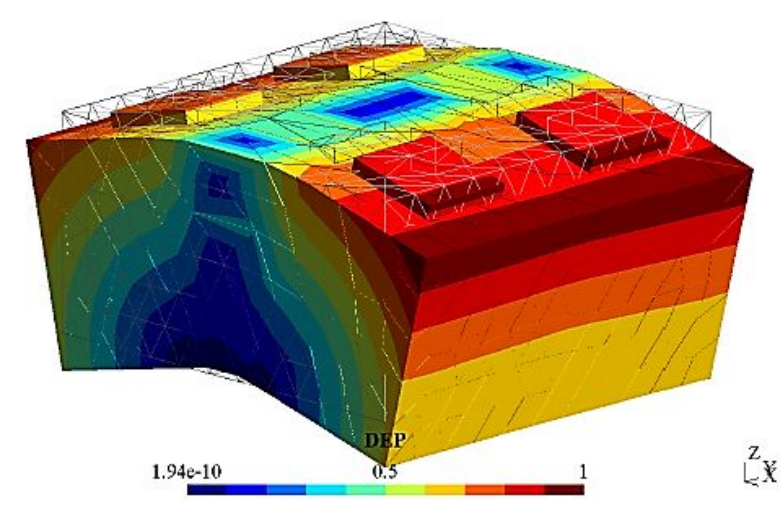

(a)

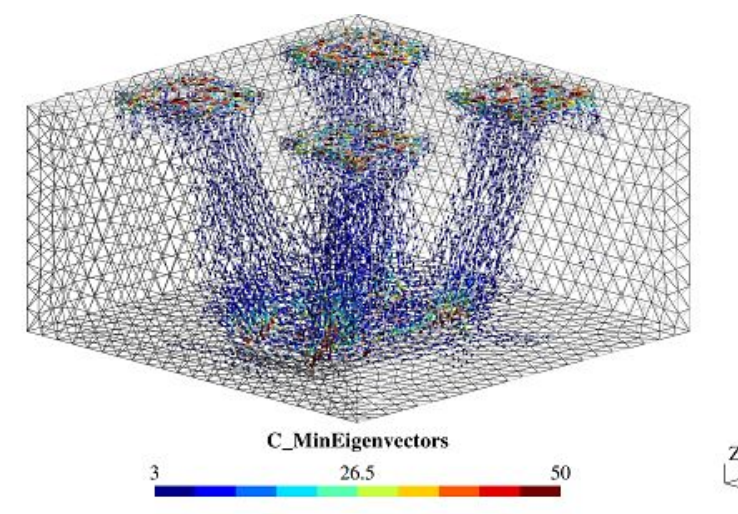

(b)

Figure 11. Optimal failure mechanism and stress field of the unreinforced bridge pier cap

\subsection{2. $T M C$ vs. Rankine criteria}

The Rankine criterion, often used in practice for modelling the resistance of plain concrete, is the simplest criterion based on the sole knowledge of the uniaxial tensile and compressive strengths of the material. It expression writes:

$$
F^{R}(\underline{\underline{\sigma}}) \leq 0 \Leftrightarrow-f_{c} \leq \sigma_{m} \leq \sigma_{M} \leq+f_{t}
$$

This criterion is exactly coincident with the $T M C$ criterion for plane stresses and $f_{t}=0$, but significantly underestimates the same criterion in the domain of compressive stresses as clearly illustrated by its intrinsic curve in the Mohr-plane drawn in Figure 12 and to be compared with 
that relative to the $T M C$ criterion shown in Figure 2. It notably appears that, unlike for the $M C T$ criterion, according to which the concrete is able to withstand any isotropic compressive stress, the Rankine criterion predicts a limited strength capacity (namely $f_{c}$ ) under this particular state of stress.

The corresponding support functions relative to a strain rate and velocity jump may be easily calculated as follows ([15],[18]):

$$
\pi^{c}(\underline{\underline{d}})=\sum_{k=1}^{3} \sup \left\{f_{t} d_{k} ;-f_{c} d_{k}\right\} ; \quad \pi^{c}(\underline{n} ; \underline{\boldsymbol{V}})=\frac{f_{t}+f_{c}}{2}|\underline{\boldsymbol{V}}|+\frac{f_{t}-f_{c}}{2} \underline{\boldsymbol{V}} \cdot \underline{n}
$$

The Rankine criterion can be implemented in both the numerical static and kinematic methods leading to the formulation of SDP optimization problems ([15], [18]) in exactly the same way as it has been done for the $T M C$ criterion.

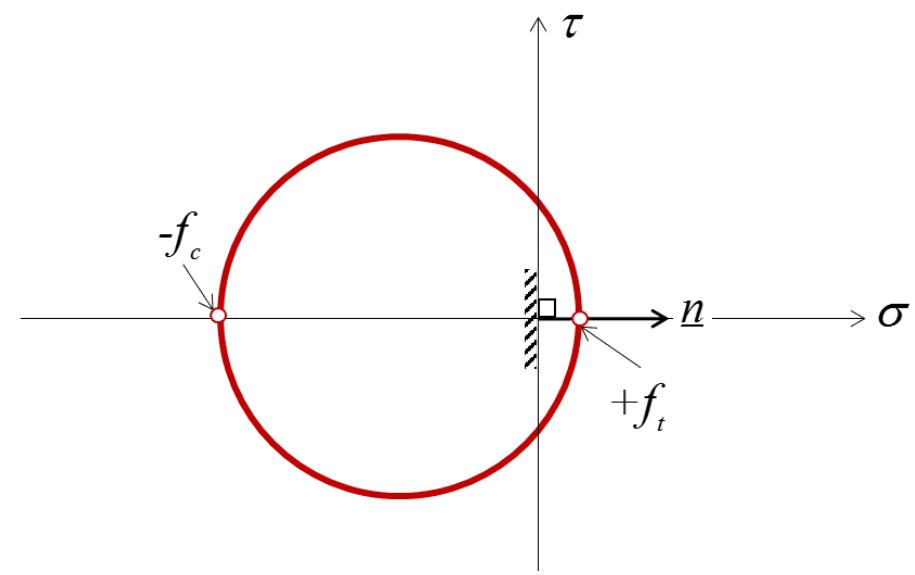

Figure 12. Intrinsic curve associated with the Rankine strength condition (24)

As a result of the analysis, Table III compares the optimized lower/upper bound estimates calculated with the Rankine and TMC criteria, adopting the same values for the uniaxial tensile and compressive strengths.

\begin{tabular}{|c|c|c|c|c|}
\cline { 2 - 5 } \multicolumn{1}{c|}{} & $P^{\mathrm{lb}}(\mathrm{MPa})$ & $P^{\mathrm{ub}}(\mathrm{MPa})$ & $\left(P^{\mathrm{lb}}+P^{\mathrm{ub}}\right) / 2(\mathrm{MPa})$ & $\Delta(\%)$ \\
\hline Rankine criterion & 3.80 & 4.00 & 3.90 & \pm 2.6 \\
\hline$T M C$ criterion & 3.89 & 4.46 & 4.17 & \pm 6.8 \\
\hline
\end{tabular}


Table III. Upper and lower bound estimates using the Rankine and the TMC criterion with the same uniaxial tensile and compressive strengths.

This table shows that the application of the numerical method leads to a more accurate estimate for the limit load when using the Rankine criterion $( \pm 2.6 \%$ instead of $\pm 6.8 \%$ for the $T M C$ criterion), but to a slightly lower value of the estimated limit load: $3.90 \mathrm{MPa}$ against $4.17 \mathrm{MPa}$ that is a relative difference of $6.5 \%$. This may be explained by the fact that the Rankine criterion tends to underestimate the strength of the plain concrete material, as compared to the $T M C$ criterion, notably in the region of compressive stresses.

\subsection{Reinforced concrete bridge pier cap.}

The same bridge pier cap as that analysed in 4.3 is now considered, with a reinforcement system made of four steel rebars located just below the loading pads as shown in Figure 13. The diameter of the reinforcing bars is equal to $\phi=3 \mathrm{~cm}$ and the uniaxial tensile-compressive resistance of the steel is taken equal to $f_{y}^{s}=400 \mathrm{MPa}$. According to the homogenization procedure described at the beginning of the paper, each steel rebar with its surrounding concrete is replaced by a homogenized square cylindrical zone of side equal to $s=0.10 \mathrm{~m}$ at any point of which the macroscopic strength condition (5) is adopted with:

$$
\sigma_{0}=\left(\pi \phi^{2} / 4\right) f_{y}^{s} / s^{2} \cong 28.2 \mathrm{MPa}
$$

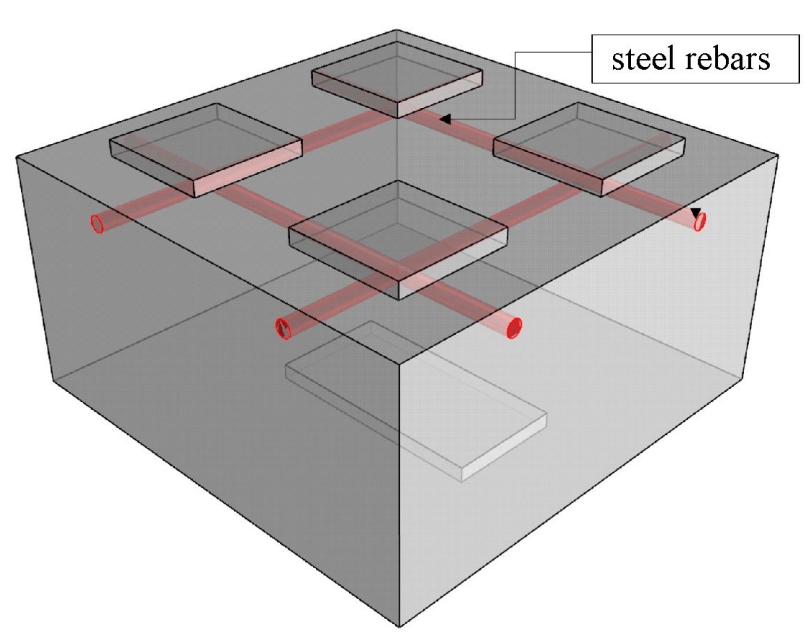

Figure 13. Bridge pier cap with its strengthening system of four steel rebars

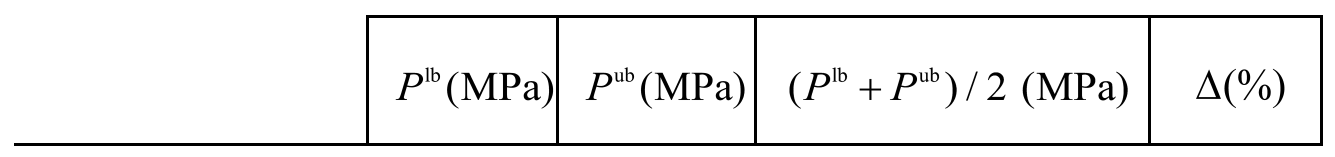




\begin{tabular}{|c|c|c|c|c|}
\hline Rankine criterion & 5.20 & 5.51 & 5.35 & \pm 2.9 \\
\hline$T M C$ criterion & 5.28 & 6.21 & 5.75 & \pm 8.1 \\
\hline
\end{tabular}

Table IV. Lower and upper bound estimates for the ultimate load bearing capacity of the reinforced bridge pier cap.

The results in terms of numerical upper and lower bound estimates of the limit pressure $P$ are reported in Table IV for the TMC as well as the Rankine criteria. The Rankine-based estimate is $5.35 \mathrm{MPa} \pm 2.9 \%$, while the $T M C$-based one is $5.75 \mathrm{MPa} \pm 8.1 \%$. From comparing with the results of Table III, one may thus conclude that the installation of the four rebars system increases the structure bearing capacity by a multiplicative factor equal to $5.35 / 3.90 \cong$ 1.373 when adopting the Rankine criterion and $6.21 / 4.17 \cong 1.376$ when using the $T M C$ criterion that is almost exactly the same value.

This also confirms that the adoption of the Rankine instead of the TMC criterion for describing the strength properties of plain concrete on the base of the same uniaxial strength characteristics, tends to underestimate the strength of concrete under multiaxial compressive stresses.

As a further illustration of the above results, Figure 14 displays the perspective and side views of the optimal stress field used in the numerical static approach. Both pictures reveal a clearly visible strut-and-tie scheme involving four compressive struts connecting the loading pads to the underlying central pier and equilibrated by four horizontal tensile ties along the rebars.
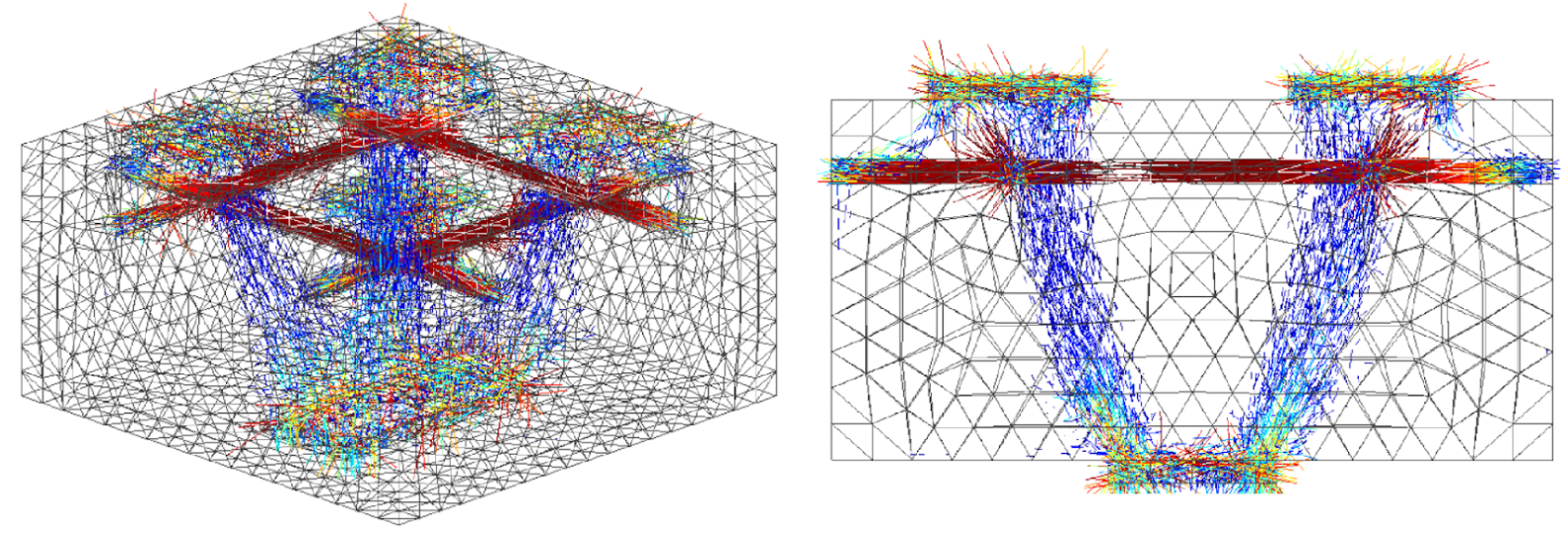

Figure 14. Optimal stress field used in the lower bound static approach

Finally, the failure mechanism associated with the optimal upper bound estimate is represented in Figure 15. 


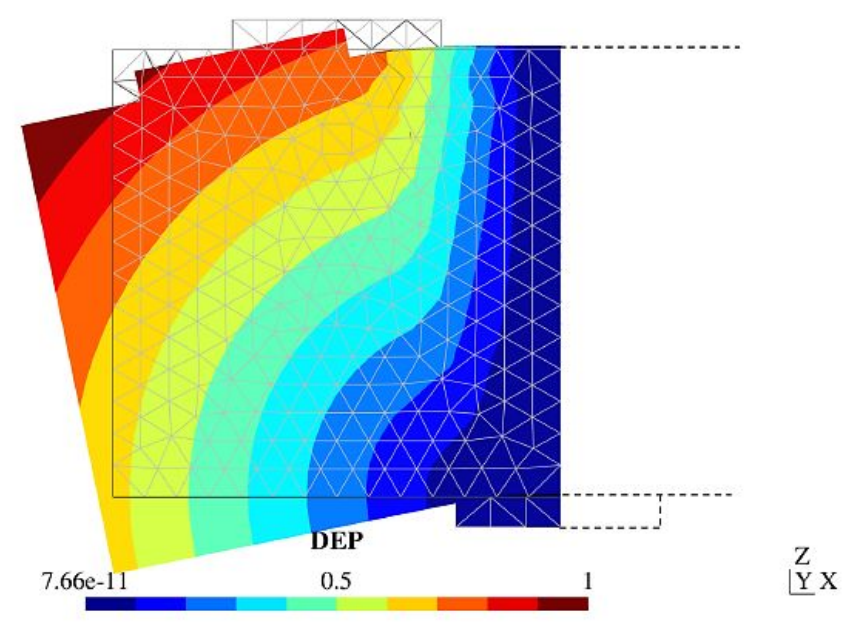

Figure 15. Optimal failure mechanism of the bridge pier cap

\section{LOAD BEARING CAPACITY OF A REINFORCED CONCRETE FOOTING}

The last example to be analysed with our approach concerns the load carrying capacity of a reinforced concrete circular slab used as a building or bridge foundation system. The results of our analysis will be compared to those established by Simões et al. [11] implementing in a semi-analytical way the kinematic approach of limit analysis/yield design.

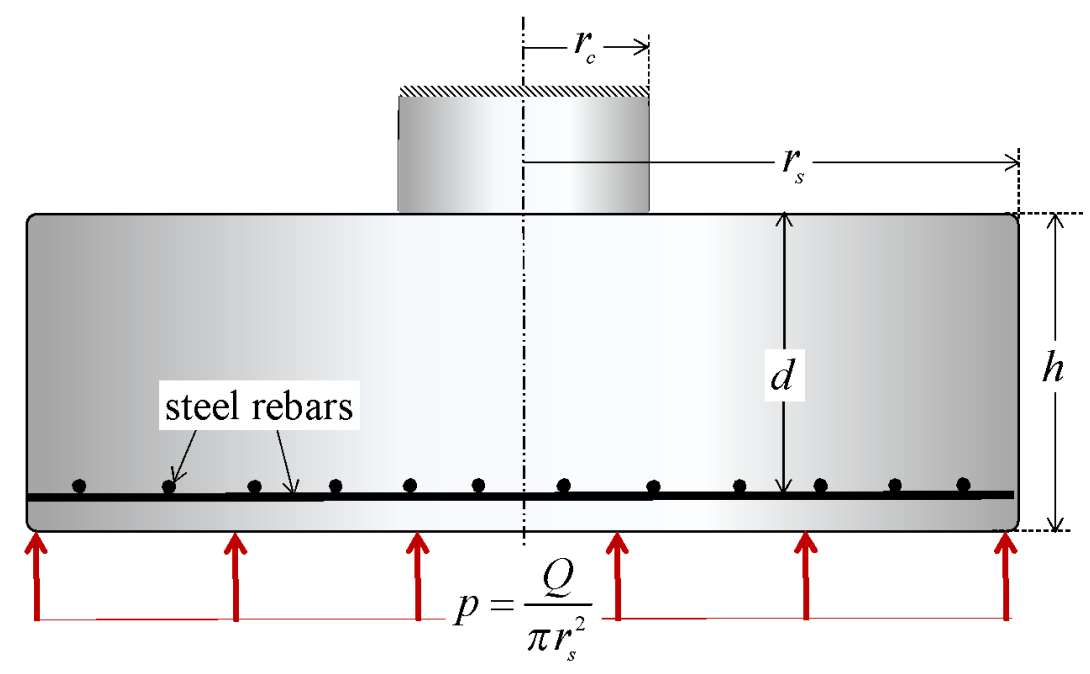

Figure 16. Geometrical characteristics and loading conditions of the reinforced concrete circular footing

The structure to be investigated is shown in Figure 16, where the notations of [11] have been adopted. The footing is a circular slab of thickness equal to $h$ and radius equal to $r_{s}$ rigidly connected in its central upper part to a column (bridge pier for instance) of radius 
$r_{c}<r_{s}$. The action of the underlying soil is simply modelled by means of a uniform pressure applied on the footing base equal to:

$$
p=\frac{Q}{\pi r_{s}^{2}}
$$

where $Q$ is the total resulting force applied by the foundation to the soil. The concrete footing has been reinforced in its lower part by a system of steel rebars placed along the radial and circumferential directions at a distance $d<h$ from the upper face of the footing, so that the problem can be dealt with as an asymmetrical limit analysis/yield design problem.

\subsection{A semi-analytical method of analysis [11].}

The semi-analytical method of analysis developed by Simões et al. [11] will now be briefly described. It is based upon the upper bound kinematic theorem of limit analysis/yield design making use of two families of simple failure mechanisms, called M1 and M2, represented in Figure 17 in any meridian plane passing through the symmetry axis, with the cylindrical coordinates $(r-z)$.

The first kind of mechanism (M1) is shown in Figure 17(a). Its trace in the meridian plane corresponds to a rigid body motion of an external part of the slab separated from its central part by a velocity discontinuity line, the instantaneous centre of rotation (ICR) being located in the upper left quarter of plane defined in the same figure. The second kind of mechanism (M2) corresponds to the situation when the ICR is located in the lower right quarter of plane shown in Figure 17(b).

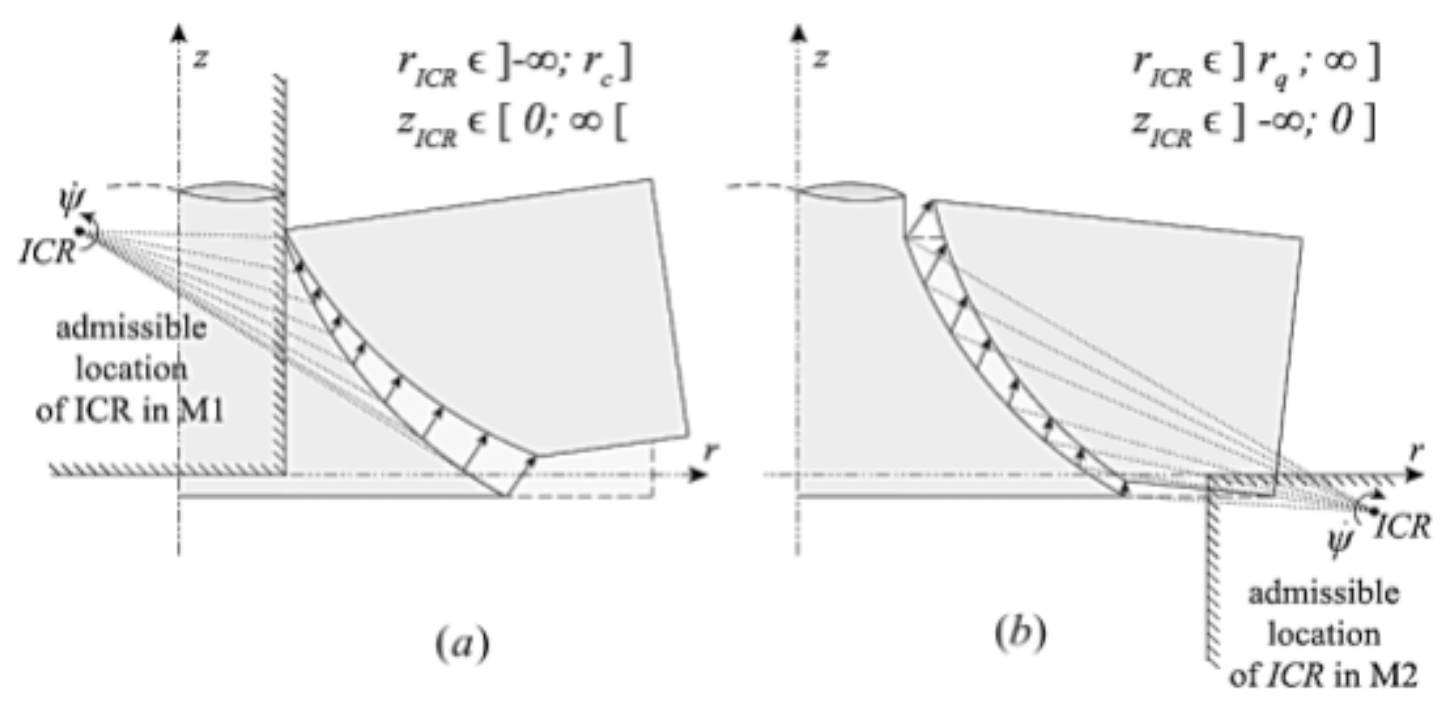


Figure 17. Failure mechanisms M1 (a) and M2 (b) used in the kinematic approach of limit analysis by [11].

It should be emphasized that, except in the particular case when the ICR is located at infinity $(r \rightarrow \pm \infty)$, which corresponds to a pure vertical translation of the rotating part, such mechanisms always involve non zero circumferential strain rates $d_{\theta \theta}$ which must be accounted for in the calculation of the maximum resisting work developed in such mechanisms, in addition to the contribution of the velocity discontinuity axisymmetric surface.

As usual, any failure mechanism of the above-described type leads to an upper bound estimate for the limit load $p^{+}=Q^{+} / \pi r_{s}^{2}$. The minimal bound is then searched with respect to the position of the ICR on the one hand, the geometry of the velocity discontinuity surface on the other hand. This minimization problem is performed numerically by discretizing the velocity discontinuity line in the meridian plane into segments.

In order to avoid such a computational time consuming procedure, Simões et al. [11] have developed simplified analytical expressions for the limit load best upper bound estimates which accurately fit the numerical estimates in the following ranges of variation of non-dimensional geometrical parameters: $r_{c} / d \leq 1.2 ;\left(r_{s}-r_{c}\right) / d \leq 3 ; r_{s} / r_{c} \leq 12$. The results of such a simplified formulation will now be compared with those of our numerical finite element approach

\subsection{Implementation of the present approach}

In the problem addressed by Simões et al. [11], the steel rebars are placed according to the layout shown in Figure 18(a) that is complying with the symmetry property about the central vertical axis. In the 3D approach developed in the present paper, the reinforcement layout will consist of a double array of perpendicular rebars as pictured in Figure 18(b), which represents a slight difference with the first configuration. The two problems may however be reasonably considered as equivalent, provided that the same global amount of reinforcement be used in the two configurations. 


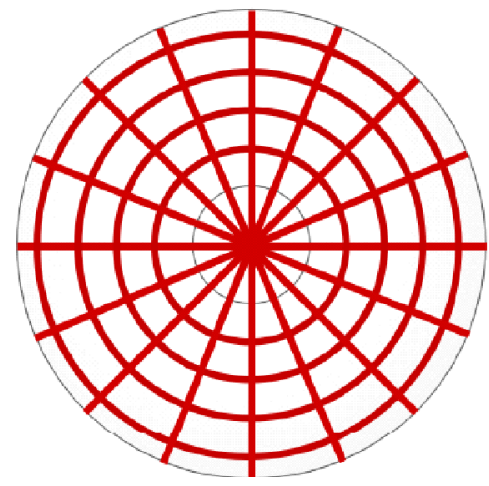

(a)

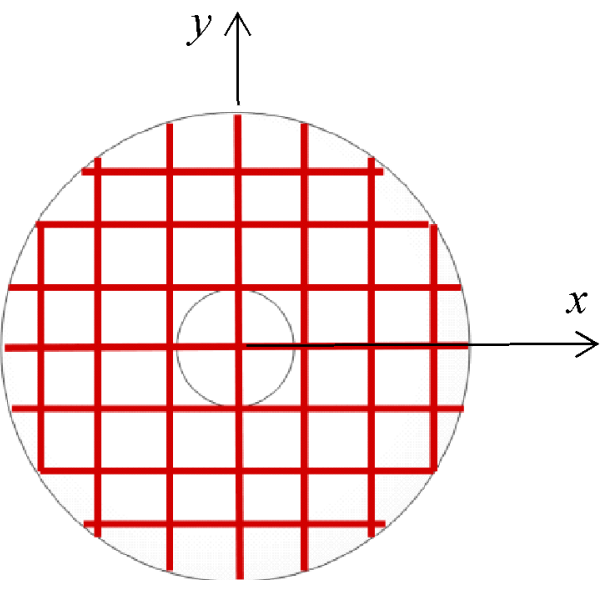

(b)

Figure 18. Reinforcement layout according to [11] (a) and the present approach (b)

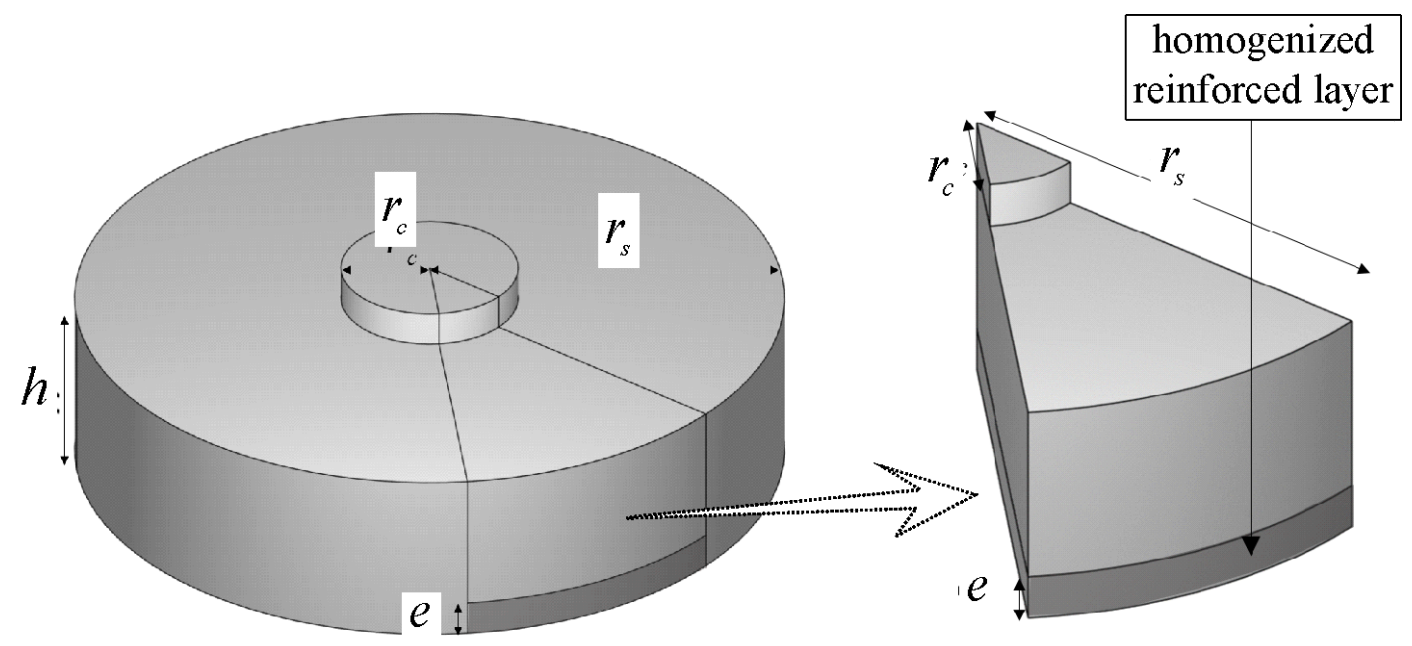

Figure $19.45^{\circ}$ angular portion of the reinforced concrete footing with its bottom homogenized layer of reinforced concrete

Owing to the symmetry properties of the second configuration, the analysis of the problem will be focused on the $1 / 8^{\text {th }}$ part of the structure shown in Figure 19. The reinforcement system placed at the base of the slab is homogenized according to a procedure extended to the case of a reinforcement in two orthogonal directions as that adopted in Figure 18(b). It can be proved (see [14] or [19] for more details) that the macroscopic strength condition at any point of the homogenized layer of concrete reinforced in directions $x$ and $y$, is given by:

$$
F^{r c}(\underline{\underline{\sigma}}) \leq 0 \Leftrightarrow\left\{\begin{array}{l}
\underline{\underline{\sigma}}=\underline{\underline{\sigma}}^{c}+\sum_{\alpha} \sigma_{\alpha}^{r} \underline{e}_{\alpha} \otimes \underline{e}_{\alpha}, \alpha=x, y \\
\text { with } F^{c}\left(\underline{\underline{\sigma}}^{c}\right) \leq 0 \text { and }-k \sigma_{\alpha 0} \leq \sigma_{\alpha}^{r} \leq+\sigma_{\alpha 0}
\end{array}\right.
$$


with

$$
\sigma_{\alpha 0}=\eta_{\alpha} f_{y}^{s}
$$

where, as in Eq.(7), $\eta_{\alpha}, \alpha=x, y$ is the reinforcement volume fraction in the direction $\alpha$ and $f_{y}^{s}$ the uniaxial strength of the constituent steel material. The corresponding support functions are:

$$
\begin{aligned}
& \pi^{r c}(\underline{\underline{d}})==\pi^{c}(\underline{\underline{d}})+\sum_{\alpha=x, y} \sigma_{0 \alpha} \sup \left\{-k d_{\alpha \alpha}, d_{\alpha \alpha}\right\} \\
& \pi^{r c}(\underline{n} ; \underline{V})=\pi^{c}(\underline{n} ; \underline{\boldsymbol{V}})+\sum_{\alpha=x, y} \sigma_{0 \alpha} \sup \left\{-k n_{\alpha} V_{\alpha}, n_{\alpha} V_{\alpha}\right\}
\end{aligned}
$$

These criteria, as well as their support functions, can be formulated as local SDP optimization problems and integrated in the global finite element SDP optimization procedure, as described in section 3 .

\subsection{Analysis of results and comparison with other calculation models}

The objective of the analysis is to investigate, for a given geometry of the footing, the influence of the rate of reinforcement defined as

$$
\omega=\rho \frac{f_{y}^{s}}{f_{c}} \text { with } \rho=\frac{A^{s}}{2 h r_{s}}
$$

(where $A^{s}$ is the total cross sectional area of the steel rebars in the meridian plane $x=0$ or $y=0$ ) on the value of the footing load bearing capacity expressed in non-dimensional form:

$$
\frac{Q^{+}}{f_{c} d^{2}}=f(\omega)
$$

The numerical bounds obtained from the 3D yield design analysis are represented by the two red coloured (upper bound) and blue coloured (lower bound) dashed curves of Figure 20. These curves have been drawn for the following values of the strength parameters:

$$
f_{c}=30 \mathrm{MPa} ; f_{t}=0,1 \mathrm{MPa} ; f_{y}^{s}=500 \mathrm{MPa}
$$

and a geometry of the footing defined by the following non-dimensional parameters:

$$
r_{c} / d=0.5 \leq 1.2 ;\left(r_{s}-r_{c}\right) / d=1.5 \leq 3 ; 2 \leq r_{s} / r_{c}=4 \leq 12
$$


This figure provides a clear evidence of the improvement of the load carrying capacity as the rate of reinforcement is increased, even though both curves seem to reach an asymptotic value beyond a rate of reinforcement equal to 0.3 .

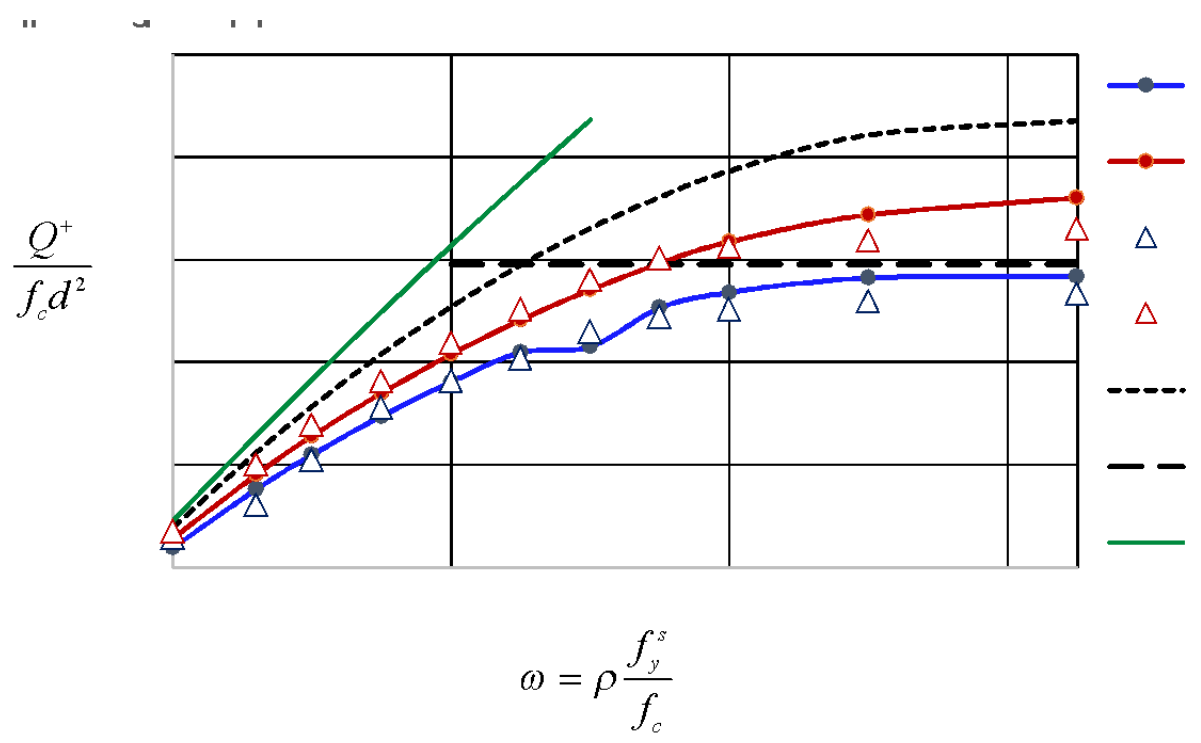

Figure 20. Comparison between different estimates of the footing load bearing capacity as functions of the rate of reinforcement

The green-coloured dashed line obtained by Simões et al. [11], which predicts an increase of the structure bearing capacity proportional to the rate of reinforcement, corresponds to its "flexural" capacity. It represents an upper bound value which overestimates all the more the exact limit load value as the rate of reinforcement increases. The grey-coloured solid curve and the horizontal black-coloured solid line correspond to the "flexural-shear" and "punching-shear" failure regimes, respectively. They have been drawn according to the above mentioned simplified analytical approximations established in [11]. It is to be observed that, while the "flexural-shear" predictions are higher that the 3D predictions, the "punching-shear" prediction (which is independent of the rate of the rate of reinforcement) lies within the interval forms by the 3D upper and lower bound estimates. It should be kept in mind however, that our $3 \mathrm{D}$ homogenized model of reinforcement is a rough approximation of the exact reinforcement layout used in [11].

In addition, upper and lower bound estimates produced by the 2D finite element limit analysis code Optum $G 2$ [20], have been reported in the same figure in the form of two series of triangular symbols. Using the axisymmetric version of this code, the bottom reinforcement system was replaced by a homogenous layer a purely cohesive material obeying a Tresca 
strength condition so as to obtain the same global amount of reinforcement. Despite such an approximation, the results obtained from this axisymmetric model are in good agreement with the $3 \mathrm{D}$ calculations, except for higher rates of reinforcement where it produces moderately lower predictions.

\subsection{Analysis of failure mechanisms}

Figure 21 visualizes the optimal velocity fields obtained from implementing the upper bound kinematic approach for three increasing rates of reinforcement: $\omega=0.05 / 0.20 / 0.375$. It deserves the following comments.

$\checkmark$ For the lowest rate of reinforcement $(\omega=0.05)$ where all calculation methods lead to very close estimates of the load bearing capacity, represented by the extreme left points of the different curves drawn in Figure 20, the observed velocity field corresponds to a pure "flexural" mechanism, in which the external part of the footing is given, in the median plane, a rigid body motion with a centre of rotation located in the vicinity of the central supported column.

$\checkmark$ Conversely, for an intermediate value of the rate of reinforcement $(\omega=0.20)$ the optimal velocity field corresponds to a "punching-shear" mechanism, that is to a uniform vertical translation of an external ring-shaped part of the footing, while the central part around the column remains motionless. It should be noted that in such a situation the velocity discontinuity across the separating surface being perpendicular to the reinforcements $\left(V_{\alpha}=0\right.$ in (36)), the contribution of the latter to the maximum resisting work reduces to zero.

$\checkmark$ Finally, for the highest value of the rate of reinforcement $(\omega=0.375:$ results points located at the right end of the curves of Figure 20), the optimal velocity field looks like a rigid block failure mechanism (in the median plane) but, contrary to the first case, with a centre of rotation located outside the footing. 

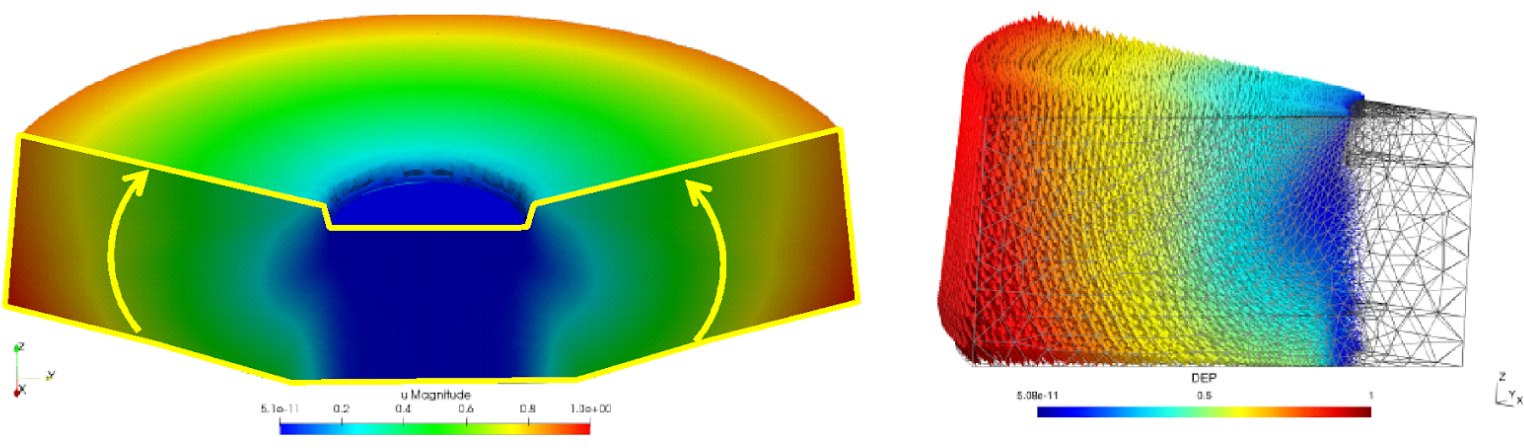

$$
\omega=0.05
$$
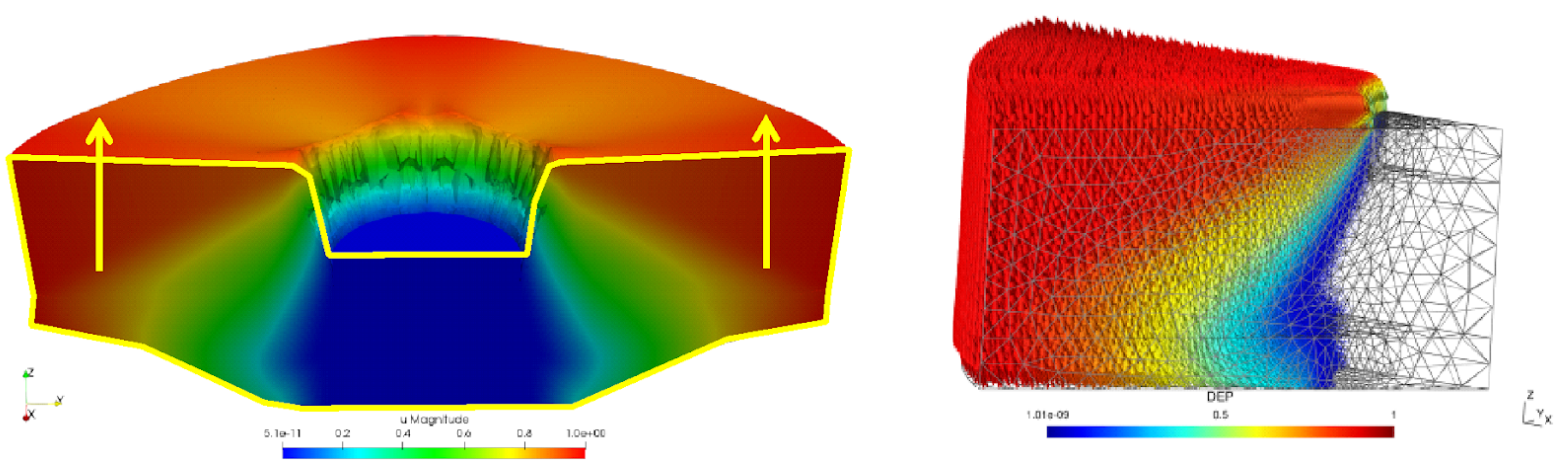

$$
\omega=0.20
$$
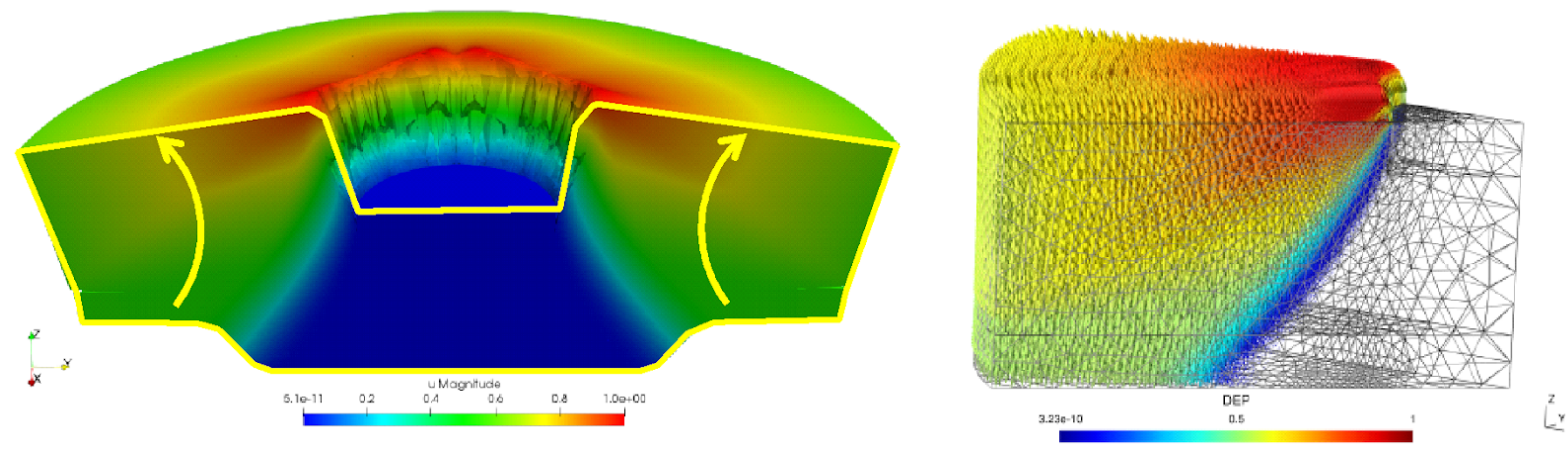

$$
\omega=0.375
$$

Figure 21. Optimal failure mechanisms obtained for three different rates of reinforcement $\omega=0.05-0.20-0.375$.

\section{CONCLUDING REMARKS}


The present paper completes the previously published one [9], which was dedicated to the numerical implementation of the lower bound static approach, by setting up a finite element computer code producing rigorous upper as well as lower bound estimates to the ultimate failure loads of fully three dimensional reinforced concrete structures, whatever the geometrical layout of the linear reinforcing elements inside the plain concrete volumes. This fully operational yield design code relies on two recently achieved significant breakthroughs:

1) The adoption of a homogenization method, developed within the yield design framework, making it possible to capture the yield strength properties of periodically distributed as well as isolated reinforcements, and incorporate them in both the static and kinematic approaches. The performance and efficiency of this method have been proved in this paper on the example of the uniformly loaded reinforced concrete beam (section 4.1).

2) The finite element discretization of both the upper and lower bound methods, leading to the formulation and numerical resolution of the corresponding non-linear optimization problems using Semidefinite Programming techniques developed by [10] for three-dimensional Mohr-Coulomb criteria.

As shown through the different applications of this paper, the results obtained from using this finite element code give upper and lower bound predictions which always remain relatively close to each other (less than $\pm 10 \%$ when taking the mean value of the upper and lower bound estimates). Moreover, comparisons with either analytical or numerical results previously obtained on some typical examples, clearly confirm the suitability of the present approach as an appropriate engineering design tool.

Two further significant improvements of the proposed method and related numerical code can be expected and achieved in the very next future:

$\checkmark$ First, the numerical performance of both the kinematic and static finite element formulations could be upgraded by resorting to adaptive mesh refinement procedures, as classically done in many finite element codes [20]. This would undoubtedly contribute to narrowing the gap between the upper and lower bounds 
and thus enhance the accuracy of the predictions, without it being necessary to increase the number of elements, keeping the computational times to reasonable levels.

$\checkmark$ On a more fundamental level, the introduction of a specific failure condition at the interface between the reinforcements and the surrounding concrete material, which appears to be more realistic than the perfect bonding condition adopted in this paper, should be considered. This would require the adoption of a so-called multiphase model, which may be perceived as an extension of the homogenization method [14], making it possible to take a specific interaction condition between the reinforcements and the concrete material, into account. 


\section{REFERENCES}

[1] Marti P. (1985). Truss models in detailing, Concr. Int., 7(12), pp. 66-73.

[2] Schlaich M., Schäfer K., Jennewein M. (1987). Toward a consistent design of structural concrete, PCI Jl., 32(3), pp. 74-150.

[3] Schlaich M., Anagnostou G. (1990). Stress Fields for Nodes of Strut-and-Tie Models, Jl. of Structural Engineering, Volume 126, N¹, pp. 13-23.

[4] Siao W. B. (1993). Strut-and-tie model for shear behaviour in deep beams and pike caps failing in diagonal splitting, ACI Struct. J., 90(4), pp. 356-363.

[5] Averbuch D., de Buhan P. (1999). Shear Design of Reinforced Concrete Deep Beams: A Numerical Approach, Jl. of Structural Engineering, Volume 125, N³, pp. 309-318.

[6] Llau A., Jason L., Dufour, F., Baroth J. (2016). Finite element modelling of 1D steel components in reinforced and pre-stressed concrete structures, Engineering Structures, 127, pp. 769-783.

[7] Nielsen M.P., Hoang L.C. (2010). Limit Analysis and Concrete Plasticity, CRC Press, Taylor \& Francis.

[8] Figueiredo M.P., Maghous S., Filho A.C. (2013). Three-dimensional finite element analysis of reinforced concrete structural elements regarded as elastoplastic multiphase media, Materials and Structures, 46, pp. 383-404.

[9] Vincent H., Arquier M., Bleyer J., de Buhan P. (2018). Yield design-based numerical analysis of three-dimensional reinforced concrete structures, Int. J. Numer. Anal. Methods Geomech., vol. 42, 18, pp. 2177-2192.

[10] Martin C.M., Makrodimopoulos A. (2008). Finite-Element Limit Analysis of Mohr-Coulomb Materials in 3D Using Semidefinite Programming, Jl. Eng. Mech., ASCE, Vol. $134, \mathrm{~N}^{\circ} 4$, pp. 339-347.

[11] Simões J., Faria D., Fernández Ruiz M., Muttoni A. (2016). Strength of reinforced concrete footings without transverse reinforcement according to limit analysis, Eng. Struct., 112, pp. 146-161.

[12] Chen W.F. (1982) Plasticity in reinforced concrete. McGraw-Hill, New York.

[13] Salençon J. (2013) Yield Design, ISTE Ltd, Wiley, London.

[14] de Buhan P., Bleyer J., Hassen G. (2017). Elastic, Plastic and Yield Design of Reinforced Structures, ISTE-Elsevier, London.

[15] Vincent H. (2018). Numerical yield design-based method for assessing the ultimate load bearing capacity of $3 D$ reinforced concrete structures, $\mathrm{PhD}$ thesis, Université Paris-Est [in French]. 
[16] The Mosek optimization software: available from http://www.mosek.com

[17] Makrodimopoulos A., Martin C.M. (2007). Upper bound limit analysis using simplex strain elements and second-order cone programming. Int. J. Numer. Anal. Methods Geomech., vol. 31, 6, pp. 835-865.

[18] http://www.optumce.com/products/optumg3

[19] Vincent H., Arquier M., Bleyer J., de Buhan P. (2018). Ultimate Limit State Design of three-dimensional reinforced concrete structures: a numerical approach. Proceedings of the conference on computational modelling of concrete and concrete structures (EURO-C 2018), Bad Hofgastein, Austria. 128.

[20] de Buhan P., Taliercio A. (1991). A homogenization approach to the yield strength of composite materials, Eur. J. of Mech., A/Solids, 10, n², pp. 129-150.

[21] http://www.optumce.com/products/optumg2/ 